\title{
Two-Dimensional Quantum-Link Lattice Quantum Electrodynamics at Finite Density
}

\author{
Timo Felser®, ${ }^{1,2,3}$ Pietro Silvi, ${ }^{4,5}$ Mario Collura ${ }^{1,2,6}$ and Simone Montangero $\circledast^{2,3}$ \\ ${ }^{1}$ Theoretische Physik, Universität des Saarlandes, D-66123 Saarbrücken, Germany \\ ${ }^{2}$ Dipartimento di Fisica e Astronomia “G. Galilei,” Università di Padova, I-35131 Padova, Italy \\ ${ }^{3}$ Istituto Nazionale di Fisica Nucleare (INFN), Sezione di Padova, I-35131 Padova, Italy \\ ${ }^{4}$ Center for Quantum Physics, and Institute for Experimental Physics, \\ University of Innsbruck, A-6020 Innsbruck, Austria \\ ${ }^{5}$ Institute for Quantum Optics and Quantum Information, \\ Austrian Academy of Sciences, A-6020 Innsbruck, Austria \\ ${ }^{6}$ SISSA-International School for Advanced Studies, I-34136 Trieste, Italy
}

(Received 13 January 2020; revised 13 July 2020; accepted 21 September 2020; published 25 November 2020)

\begin{abstract}
We present an unconstrained tree-tensor-network approach to the study of lattice gauge theories in two spatial dimensions, showing how to perform numerical simulations of theories in the presence of fermionic matter and four-body magnetic terms, at zero and finite density, with periodic and open boundary conditions. We exploit the quantum-link representation of the gauge fields and demonstrate that a fermionic rishon representation of the quantum links allows us to efficiently handle the fermionic matter while finite densities are naturally enclosed in the tensor network description. We explicitly perform calculations for quantum electrodynamics in the spin-one quantum-link representation on lattice sizes of up to $16 \times 16$ sites, detecting and characterizing different quantum regimes. In particular, at finite density, we detect signatures of a phase separation as a function of the bare mass values at different filling densities. The presented approach can be extended straightforwardly to three spatial dimensions.

DOI: $10.1103 /$ PhysRevX.10.041040

Subject Areas: Computational Physics,

Particles and Fields, Quantum Physics
\end{abstract}

\section{INTRODUCTION}

Recent progress in quantum simulations is paving the way for the possibility of studying high-energy physics phenomena with tools developed in low-energy quantum physics [1-13]. In the Standard Model, forces are mediated through gauge fields; thus, gauge-invariant field theoriese.g., quantum electrodynamics (QED) for the Abelian case or quantum chromodynamics (QCD) for the non-Abelian scenario - are fundamental building blocks to our understanding of all microscopic processes ruling the dynamics of elementary particles $[14,15]$. When discretizing the gauge theories, the dynamical gauge variables obey a lattice formulation of the original quantum field theory, which is referred to as a lattice gauge theory (LGT) $[16,17]$. LGTs encode many-body interactions satisfying exact constraints, encoding a lattice-discretized version of the local gauge invariance, e.g., in QED, the Gauss law $\nabla \cdot E=4 \pi \rho$. Many of the collective phenomena arising from these theories, including the phase diagram, have yet to be fully

Published by the American Physical Society under the terms of the Creative Commons Attribution 4.0 International license. Further distribution of this work must maintain attribution to the author(s) and the published article's title, journal citation, and DOI. characterized [18], especially for higher spatial dimensions at finite charge density.

Possibly the most successful tools to investigate LGTs are Monte Carlo simulations based on lattice formulations [16,19-23]. However, the Monte Carlo approach suffers from the infamous sign problem for complex actions, e.g., at finite fermion density (matter-antimatter unbalance), which naturally arises in LGTs [13,24]. Another very promising alternative to simulate lattice gauge theories is based on tensor network (TN) methods. They have already shown significant capabilities in describing many condensed matter and chemistry problems and for studying lattice gauge theories in one spatial dimension [12,25-31, 31-41]. So far, very few attempts have been made to capture the phase properties (e.g., at zero temperature) of a lattice analogue of an Abelian gauge theory in higher spatial dimensions $[4,42-50]$, none of them in the presence of fermionic matter at finite density.

In this work, we fill this gap and develop a computationally tractable Hamiltonian formulation of low-energy QED in two spatial dimensions. We show that TN states allow for an accurate representation of its many-body ground state, thus allowing us to identify the different regimes and effectively test the response of the system to a finite density of charge. The study of lattice gauge Hamiltonians at finite chemical potential is, in general, 
out of reach for Monte Carlo-based techniques [13,24]: Here, we show that by using an unconstrained tree tensor network (TTN) [51] and the quantum-link formalism of lattice gauge theories [2,52-55], we can face this highly nontrivial setup. The techniques developed in this paper not only provide the basic ingredients for an efficient calculation of the phase diagram of simple lattice gauge models, but they can also be extended to more complex theories and higher dimensions.

We demonstrate the effectiveness of the presented approach by focusing on the low-energy properties, both at zero and finite charge density, of a two-dimensional lattice quantum-link theory with $U(1)$ gauge symmetry. Specifically, we investigate a model involving (spinless, flavorless) Kogut-Susskind matter fermions [16,21] and $U(1)$ electromagnetic gauge fields, truncated to a spin- $S$ compact representation. Hereafter, we set $S=1$, the smallest representation where all Hamiltonian terms are nontrivial. The calculations for higher spin representations are numerically demanding but straightforward. We investigate the (zero-temperature) phase diagram in the zero global charge scenario without and with finite magnetic coupling. We observe that both magnetic and electric Hamiltonian terms, separately, hinder the creation of a charge-crystal configuration, which emerges at large negative bare masses. However, when electric and magnetic terms are mutually frustrated, the charge crystal is restored. Moreover, we study the ground state in the presence of a finite charge density, which we can directly control in the TN ansatz state. Small charge densities impact the zerocharge phases as follows: In the vacuum regime, charges aggregate at the system (open) boundaries, suggesting the existence of a spatial phase separation between the bulk and the boundaries; this scenario is reminiscent of the classical electrodynamics properties of a perfect conductor, where $\nabla \cdot E=0$ in the bulk and the excess of charge is redistributed on the outer surface of the conductor. On the contrary, the charge-crystal regime, which is full of matter or antimatter, is characterized by a homogeneous delocalization of the charge hole, resulting in a quasiflat charge distribution in the bulk and therefore reminiscent of a plasma phase [56].

Finally, we stress that the quantum-link formulation provides the ideal tools to establish a connection between LGTs and atomic lattice experiments [57,58]. In this framework, the dynamical gauge fields are usually represented by spin degrees of freedom, which have a natural mapping to typical condensed-matter models, like Hubbard Hamiltonians or locally constrained Ising-like Hamiltonians. These models can be engineered with cold atoms in optical lattices $[7,11]$, or within the very promising experimental setups involving Rydberg atom chains $[59,60]$, and they can be straightforwardly numerically simulated with the presented techniques to verify and benchmark the experimental results and to carefully and quantitatively compare the limits, the precision, and the efficiencies of the classical and quantum simulations.

The paper is structured as follows: In Sec. II, we present the 2D lattice gauge Hamiltonian and its quantum-link formulation in terms of the gauge-field spin-1 compact representation. We also give some technical details of the tensor network numerical simulations. In Sec. III, we focus on the ground-state properties in the zero-charge sector: We explore the phase space of the model by varying the mass and the electric coupling; we then analyze the effect of a finite magnetic coupling. Section IV is devoted to studying the equilibrium properties at finite charge density. We exploit TTN techniques to investigate how the charges redistribute all over the lattice, depending on the Hamiltonian couplings. Finally, we draw our conclusions in Sec. V and give additional supplementary technical details in the Appendixes.

\section{MODEL AND METHODS}

We consider a field theory on a $2 \mathrm{D}$ square lattice with $U(1)$ local gauge symmetry. The sites of a finite $L \times L$ square lattice host the matter field, while the quantum gauge field lives on the lattice links, with open boundary conditions. Following the Kogut-Susskind (staggered) formulation [16,21], the discretization of the matter field is performed by introducing a staggered fermionic field, whose positive energy solutions lie on the even sites and whose negative ones lie on the odd sites. The matter field is thus described by spinless, flavorless Dirac fermions, whose operator algebra satisfies the usual canonical anticommutation relations $\left\{\hat{\psi}_{x}, \hat{\psi}_{x^{\prime}}^{\dagger}\right\}=\delta_{x, x^{\prime}}$. In particular, in the even sublattice, particles represent fermions with electric charge $+q$ ("positrons"), while in the odd sublattice, holes represent antifermions with electric charge $-q$ ("electrons"). Here, a lattice site $x$ labels a 2D coordinate $x \equiv(i, j)$, and the parity $p_{x} \equiv(-1)^{x}=(-1)^{i+j}$ of a site, distinguishing the two sublattices, is well defined on the square lattice (see Fig. 1).

The gauge field is defined on the lattice links, and its algebra is constructed by the electric-field operator $\hat{E}_{x, \mu}=\hat{E}_{x, \mu}^{\dagger}$ and its associated parallel transporter $\hat{U}_{x, \mu}$, which is unitary, $U_{x, \mu} U_{x, \mu}^{\dagger}=\mathbb{1}$, and satisfies $\left[\hat{E}_{x, \mu}, \hat{U}_{y, \nu}\right]=\delta_{x, y} \delta_{\mu, \nu} \hat{U}_{x, \mu}$. Here, $\mu(\nu)$ represents the positive unit lattice vector in one of the two orthogonal directions, namely, $\mu_{x} \equiv(1,0)$ and $\mu_{y} \equiv(0,1)$; thus, $(x, \mu)$ uniquely defines a link. For comfort of notation, we also allow (technically redundant) negative unit lattice vectors $-\mu_{x}$ and $-\mu_{y}$, with the convention $\hat{E}_{x+\mu,-\mu}=-\hat{E}_{x, \mu}$, and in turn $\hat{U}_{x+\mu,-\mu}=\hat{U}_{x, \mu}^{\dagger}$. With such definitions, apart from a rescaling due to the lattice spacing regularization [16,21], the two-dimensional lattice QED Hamiltonian, including a magnetic plaquette term, reads 


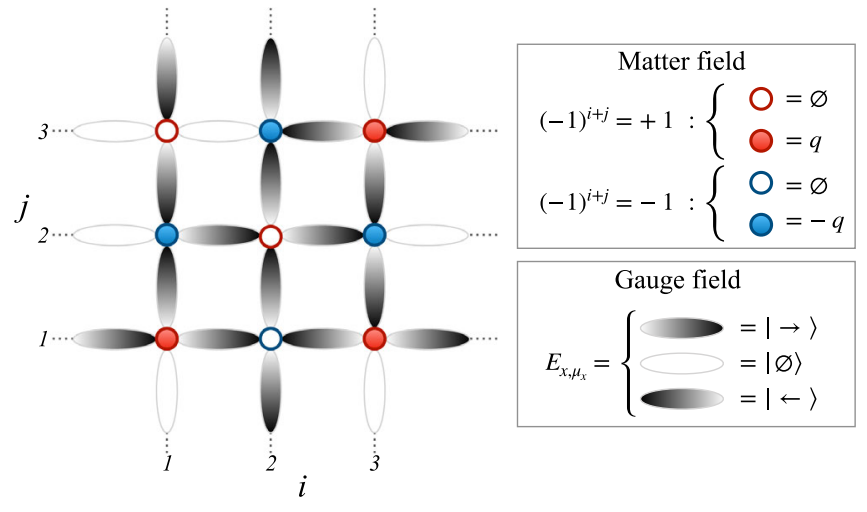

FIG. 1. Sketch of the 2D lattice gauge theory in the spin-1 representation. The cartoon of the square lattice shows a specific gauge-invariant configuration of the matter and gauge fields with zero total charge (three particles and three antiparticles). Staggered fermions represent matter and antimatter fields on a lattice bipartition: On the even (odd) bipartition, a full red (blue) site represents a particle (antiparticle). The gauge-field points in the positive direction of the color gradient.

$$
\begin{aligned}
\hat{H}= & -t \sum_{x, \mu}\left(\hat{\psi}_{x}^{\dagger} \hat{U}_{x, \mu} \hat{\psi}_{x+\mu}+\text { H.c. }\right) \\
& +m \sum_{x}(-1)^{x} \hat{\psi}_{x}^{\dagger} \hat{\psi}_{x}+\frac{g_{e}^{2}}{2} \sum_{x, \mu} \hat{E}_{x, \mu}^{2} \\
& -\frac{g_{m}^{2}}{2} \sum_{x}\left(\hat{U}_{x, \mu_{x}} \hat{U}_{x+\mu_{x}, \mu_{y}} \hat{U}_{x+\mu_{y}, \mu_{x}}^{\dagger} \hat{U}_{x, \mu_{y}}^{\dagger}+\text { H.c. }\right),
\end{aligned}
$$

where $\mu$ in $\left\{\mu_{x}, \mu_{y}\right\}$ are the unit vectors of the square lattice. The first term in Eq. (1) provides the minimal coupling between gauge and matter fields associated with the coupling strength $t$. It describes a process of particleantiparticle pair creation or annihilation, where the parallel transporter operator guarantees that the local gauge symmetries are not violated. The second term in the Hamiltonian represents the energy associated with the fermionic bare mass, and it appears as a staggered chemical potential according to the Kogut-Susskind prescription. For numerical purposes, it has been redefined by adding an overall constant $m L^{2} / 2$, thus replacing $(-1)^{x} \hat{\psi}_{x}^{\dagger} \hat{\psi}_{x} \rightarrow \delta_{x, e} \hat{\psi}_{x}^{\dagger} \hat{\psi}_{x}+\delta_{x, o} \hat{\psi}_{x} \hat{\psi}_{x}^{\dagger}$ (see Appendix C). Thus, a filled local state in the even sublattice costs positive energy $m$ and carries charge $q$; otherwise, when an odd site is empty, the energy cost is still $m$, but it corresponds to having an antiparticle (a hole) with charge $-q$. The last two terms contribute to the gauge-field dynamics: The electric part, with coupling $g_{e}$, is completely local. The magnetic part, with coupling $g_{m}$ instead, is constructed by considering the smallest Wilson loopthe product of the parallel transporter $\hat{U}_{x, \mu}$ in a closed loop - the size of a plaquette. Its name is related to the fact that it generates the magnetic contribution to the energy density in the continuum limit.
The LGT Hamiltonian $\hat{H}$ commutes with the local Gauss law generators (in units of $q$ )

$$
\hat{G}_{x}=\hat{\psi}_{x}^{\dagger} \hat{\psi}_{x}-\frac{1-p_{x}}{2}-\sum_{\mu} \hat{E}_{x, \mu},
$$

where the unit lattice vector $\mu$ in the sum runs in $\left\{ \pm \mu_{x}, \pm \mu_{y}\right\}$, while $p_{x}=(-1)^{x}$ is, again, the lattice site parity. In addition, the model exhibits a $U(1)$ global symmetry-namely, the conservation of the total charge $\hat{Q}=\sum_{x}\left[\hat{\psi}_{x}^{\dagger} \hat{\psi}_{x}-\left(1-p_{x} / 2\right)\right]=-\left(L^{2} / 2\right)+\hat{N}$, equivalent (apart from a constant) to the number conservation $\hat{N}=$ $\sum_{x} \hat{\psi}_{x}^{\dagger} \hat{\psi}_{x}$ of Kogut-Susskind matter fermions. As a consequence of the convention, using $\hat{E}_{x,-\mu}=-\hat{E}_{x-\mu, \mu}$, the sum of all four terms of the gauge field around the lattice site $x$ corresponds to the outgoing electric flux, i.e., $\sum_{\mu} \hat{E}_{x, \mu}=E_{x, \mu_{x}}+E_{x, \mu_{y}}-E_{x-\mu_{x}, \mu_{x}}-E_{x-\mu_{y}, \mu_{y}}$. The gaugeinvariant Hilbert space is thus given by all states $|\Phi\rangle$ satisfying $\hat{G}_{x}|\Phi\rangle=0$ at every site $x$. As each electric-field degree of freedom is shared by two Gauss generators $G_{x}$, the generators themselves overlap, and projecting onto the gauge-invariant subspace becomes a nonlocal operation. Only for 1D lattice QED, or the lattice Schwinger model [19], it is possible to integrate out the gauge variables and work with the matter field only (albeit with long-range interactions) [61]. However, in two dimensions, a given (integer occupation) realization of the matter fermions does not fix a unique gauge-field configuration, thus requiring explicit treatment of the gauge fields as quantum variables. A numerically relevant complication, related to the standard Wilson formulation of lattice gauge theories, arises from the gauge-field algebra, $[\hat{E}, \hat{U}]=\hat{U}$, with $\hat{E}=\hat{E}^{\dagger}$ and $\hat{U} \hat{U}^{\dagger}=\hat{U}^{\dagger} \hat{U}=\mathbb{1}$, whose representations are always infinite dimensional. Simply put, if a representation contains the gauge-field state $|\alpha\rangle$, such that $\hat{E}|\alpha\rangle=\alpha|\alpha\rangle$ with $\alpha \in \mathbb{R}$, then the states $|\alpha \pm 1\rangle=\hat{U}^{ \pm 1}|\alpha\rangle$ belong to the representation as well. By induction, the representation must contain all the states $|\alpha+\mathbb{N}\rangle$, which are mutually orthogonal as distinct eigenstates of $\hat{E}$; thus, the representation space dimension is at least countably infinite.

In order to make the Hamiltonian numerically tractable via tensor network methods, we need to truncate the local gauge-field space to a finite dimension. For bosonic models, this truncation is typically done by introducing an energy cutoff and eliminating states with single-body energy density beyond it, while checking a posteriori the introduced approximation. Similarly, for $U(1)$ lattice gauge theories, we truncate the electric field according to the quantum-link model formulation. Specifically, the gauge fields are substituted by spin operators, namely, $\hat{E}_{x, \mu}=$ $\left(\hat{S}_{x, \mu}^{z}+\alpha\right)$ and $\hat{U}_{x, \mu}=\hat{S}_{x, \mu}^{+} / s$, such that $\hat{E}$ is still Hermitian and the commutation relation $\left[\hat{E}_{x, \mu}, \hat{U}_{y, \nu}\right]=\delta_{x, y} \delta_{\mu, \nu} \hat{U}_{x, \mu}$ is preserved [2]; however, $\hat{U}$ is no longer unitary for any finite 
spin- $s$ representation $|\hat{\boldsymbol{S}}|^{2}=s(s+1) \mathbb{1}$. The original algebra is then restored in the large spin limit $s \rightarrow \infty$, for any background field $\alpha \in \mathbb{R}$. Similar truncation strategies, based on group representations, can be applied to nonAbelian gauge theories as well $[35,62]$. In the following, we make use of the spin-1 representation $(s=1)$, under zero background field $\alpha=0$, which captures reasonably well the low-energy physics of the theory, especially in the parameter regions wherein the ground state is characterized by small fluctuations above the bare vacuum. Here, $s=1$ is the smallest spin representation exhibiting a nontrivial electric energy contribution. In fact, for $s=1 / 2$, we have that $\hat{E}_{x, \mu}^{2} \propto\left(\sigma_{x, \mu}^{z}\right)^{2}=\mathbb{1}$ is simply a constant in the Hamiltonian; thus, $g_{e}^{2}$ plays no role. In $1 \mathrm{D}$, evidence suggest that truncated gauge representations converge rapidly to the continuum theory, e.g., in the Schwinger model $[37,63,64]$, reinforcing the quantitative validity of the results obtained in the simplified model. Deviations between the truncated and the full-fledged lattice theory are expected to arise when $g_{m}^{2}$ is the dominant coupling, as we show for a $2 \times 2$ example in Appendix I.

Let us mention that, in the formulation of the lattice QED implemented on our numerical algorithms, Eq. (1), we consider the respective couplings of the various Hamiltonian terms, namely, $t, m, g_{e}^{2}$, and $g_{m}^{2}$, as independent, dimensionless parameters. In this way we have a practical advantage in our numerical interface which allows us to treat the Hamiltonian terms on equal footage, and, in what follows, we set the energy scale as $t=1$. However, we stress that in the original Hamiltonian formulation of lattice QED [16,21], these couplings are mutually related as $t=(1 / a), m=m_{0}, g_{e}^{2}=\left(g^{2} / a\right), g_{m}^{2}=\left(8 / g^{2} a\right)$, where $g$ is the coupling constant of QED, $m_{0}$ is the matter-field bare mass, and $a$ is the lattice spacing of the lattice discretization. In this sense, physical realizations of lattice QED only depend on two actual parameters: $m^{\prime}=m_{0} a>0$ and $g^{2}>0$. Nevertheless, in this work, we aim to highlight that our numerical simulations are not limited to these physical scenarios, and we keep our effective couplings independent and not bound to positive values. We leave a more detailed convergence analysis along the physical regimes to future work, along the lines of similar studies already presented for 1D systems [13].

\section{A. Spin-1 compact representation of $U(1)$}

In the spin-1 representation, the electric-field operator allows three orthogonal states for the electric flux (in units of the charge $q$ ), graphically represented in Fig. 1. For a horizontal link $\left(x, \mu_{x}\right)$, we write the eigenbasis of $E_{x, \mu_{x}}$ as

$$
\hat{E}_{x, \mu_{x}}|\rightarrow\rangle=+|\rightarrow\rangle, \quad \hat{E}_{x, \mu_{x}}|\varnothing\rangle=0, \quad \hat{E}_{x, \mu_{x}}|\leftarrow\rangle=-|\leftarrow\rangle,
$$

on which the parallel transporter acts as $\hat{U}_{x, \mu_{x}}|\rightarrow\rangle=0$, $\hat{U}_{x, \mu_{x}}|\varnothing\rangle=|\rightarrow\rangle$ and $\hat{U}_{x, \mu_{x}}|\leftarrow\rangle=|\varnothing\rangle$, and analogously
$\hat{E}_{x+\mu_{x},-\mu_{x}}|\rightarrow\rangle=-|\rightarrow\rangle$. A similar set of states can be defined in the vertical links $\left(x, \mu_{y}\right)$, such that $\hat{E}_{x, \mu_{y}}|\uparrow\rangle=|\uparrow\rangle, \hat{E}_{x, \mu_{y}}|\varnothing\rangle=0$, and $\hat{E}_{x, \mu_{y}}|\downarrow\rangle=-|\downarrow\rangle$.

In this work, we introduce an algebraic technique, similar to the rishon representations common in quantum-link models [2,52-55], which has the advantage of automatically accounting for the Gauss law, while carefully reproducing the anticommutation relations of the matter fermions without resorting to Jordan-Wigner string terms (see next section). This strategy relies on splitting the gauge-field space on each link $(x, \mu)$ into a pair of three hardcore fermionic modes, defined later. We say that each mode in this pair "belongs" to either of the sites sharing the link, in this case, $x$ and $x+\mu$.

Thus, we write $\hat{U}_{x, \mu}=\hat{\eta}_{x, \mu} \hat{\eta}_{x+\mu,-\mu}^{\dagger}$, where the three hardcore fermionic operators $\hat{\eta}$ satisfy $\hat{\eta}_{x, \mu}^{3}=0$ (while $\hat{\eta}_{x, \mu}^{2} \neq 0$ ) and anticommute at different positions $\left\{\hat{\eta}_{x, \mu}, \hat{\eta}_{y, \nu}^{(\dagger)}\right\}=0$, for $x \neq y$ or $\mu \neq \nu$. Moreover, these new modes obey anticommutation relations with the matter field as well, i.e., $\left\{\hat{\eta}_{x, \mu}^{(\dagger)}, \hat{\psi}_{y}^{(\dagger)}\right\}=0$. To explicitly build this three hardcore fermionic mode $\hat{\eta}_{x, \mu}$, we use two subspecies of Dirac fermions $\hat{a}_{x, \mu}$ and $\hat{b}_{x, \mu}$, such that

$\hat{\eta}_{x, \mu}^{\dagger}=\hat{n}_{x, \mu}^{a} \hat{b}_{x, \mu}^{\dagger}+\left(1-\hat{n}_{x, \mu}^{b}\right) \hat{a}_{x, \mu}^{\dagger}, \quad\left(\hat{\eta}_{x, \mu}^{\dagger}\right)^{2}=\hat{b}_{x, \mu}^{\dagger} \hat{a}_{x, \mu}^{\dagger}$,

where $\hat{n}_{x, \mu}^{a}=\hat{a}_{x, \mu}^{\dagger} \hat{a}_{x, \mu}$ and $\hat{n}_{x, \mu}^{b}=\hat{b}_{x, \mu}^{\dagger} \hat{b}_{x, \mu}$ are the occupation number operators for each subspecies. This construction provides the local algebra

$$
\left[\hat{\eta}_{x, \mu}, \hat{\eta}_{x, \mu}^{\dagger}\right]=1-\hat{n}_{x, \mu}^{a}-\hat{n}_{x, \mu}^{b}
$$

and only grants access to the three-dimensional subspaces for each three hardcore fermion mode, spanned by the following three states:

$$
|0\rangle_{x, \mu}, \quad|1\rangle_{x, \mu}=\hat{a}_{x, \mu}^{\dagger}|0\rangle_{x, \mu}, \quad|2\rangle_{x, \mu}=\hat{b}_{x, \mu}^{\dagger} \hat{a}_{x, \mu}^{\dagger}|0\rangle_{x, \mu},
$$

where $|0\rangle_{x, \mu}$ is the Dirac vacuum of both subspecies, i.e., $a_{x, \mu}|0\rangle_{x, \mu}=b_{x, \mu}|0\rangle_{x, \mu}=0$. The state $\hat{b}_{x, \mu}^{\dagger}|0\rangle_{x, \mu}$ is disconnected from the other three and thus projected away. Such a "half-link" local subspace is joined with a similar construction $\hat{a}_{x+\mu,-\mu}^{\dagger}$ and $\hat{b}_{x+\mu,-\mu}^{\dagger}$ on the other half of the link; thus, the pair defines the link space, and $\hat{E}_{x, \mu}$ will be diagonal in the occupation basis. While, in principle, a full link space is $3 \times 3=9$ dimensional, we can now exploit the following symmetry:

$$
\hat{L}_{x, \mu}=\hat{n}_{x, \mu}^{a}+\hat{n}_{x, \mu}^{b}+\hat{n}_{x+\mu,-\mu}^{a}+\hat{n}_{x+\mu,-\mu}^{b},
$$

which counts the total number of fermions in each link and is a conserved quantity since $\left[\hat{L}_{x, \mu}, \hat{E}_{x, \mu}\right]=\left[\hat{L}_{x, \mu}, \hat{U}_{x, \mu}\right]=0$. By working in the subspace with two fermions per link, 


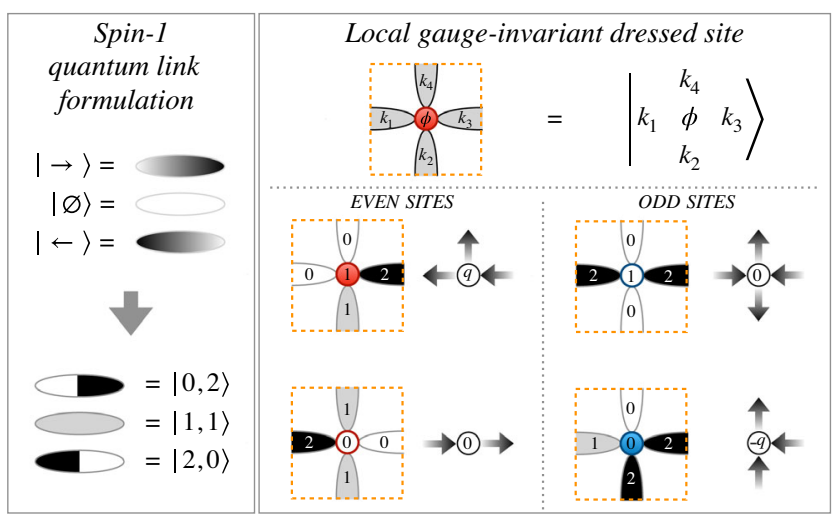

FIG. 2. Mapping of the gauge-field states in the spin-1 representation to the fermionic Fock states. Each half-link is constructed by employing two species of Dirac fermions. The link symmetry formally reduces the total number of states to only the three allowed states with two fermions. We therefore construct the local gauge-invariant dressed site by gluing each single matter site together with its neighboring half-links. In the four examples, notice how the quantum number $\phi=1$ represents the presence (absence) of a charge (anticharge) in the even (odd) sites.

$\hat{L}_{x, \mu}=2$, we reduce the link space to dimension three, and we can restore the desired algebra. First, we write the occupation basis (see also Fig. 2) as

$$
\begin{aligned}
|\rightarrow\rangle & =-|0,2\rangle=\hat{a}_{x+\mu,-\mu}^{\dagger} \hat{b}_{x+\mu,-\mu}^{\dagger}|0\rangle_{x, \mu}|0\rangle_{x+\mu,-\mu}, \\
|\varnothing\rangle & =|1,1\rangle=\hat{a}_{x, \mu}^{\dagger} \hat{a}_{x+\mu,-\mu}^{\dagger}|0\rangle_{x, \mu}|0\rangle_{x+\mu,-\mu}, \\
|\leftarrow\rangle & =|2,0\rangle=\hat{b}_{x, \mu}^{\dagger} \hat{a}_{x, \mu}^{\dagger}|0\rangle_{x, \mu}|0\rangle_{x+\mu,-\mu},
\end{aligned}
$$

so that $\hat{U}_{x, \mu}$ acts correctly, i.e., where the minus sign in the first equation ensures $\hat{U}_{x, \mu}|\varnothing\rangle=|\rightarrow\rangle$ and $\hat{U}_{x, \mu}^{\dagger}|\rightarrow\rangle=|\varnothing\rangle$. Then, we express the electric-field operator as the unbalance of fermions between the two halves of a link, precisely

$$
\hat{E}_{x, \mu}=\frac{1}{2}\left(\hat{n}_{x+\mu,-\mu}^{a}+\hat{n}_{x+\mu,-\mu}^{b}-\hat{n}_{x, \mu}^{a}-\hat{n}_{x, \mu}^{b}\right),
$$

implementing the correct action of $E_{x, \mu}$. It is worth mentioning that this formulation can be extended to higher spin- $j$ representations, where each link becomes a pair of $(2 j+1)$ hardcore fermions.

\section{B. Local gauge-invariant dressed sites}

One of the common issues while working with a lattice gauge theory, even in the compact representation of the electric field, is to properly identify the gauge-invariant Hilbert space. Because of the overlapping of the Gauss law generators $\hat{G}_{x}$, the identification of the correct local basis is highly nontrivial, especially for dimensions higher than one [26].
Using the three-hardcore-fermion pairs language gives us a shortcut to this issue. In fact, we are able to recast the Gauss law generators as nonoverlapping operators, at the price of enforcing the link constraint $\left(\hat{L}_{x, \mu}-2\right)|\Phi\rangle=0$. Using this constraint, we can rewrite the electricfield operator in Eq. (8), taking only the fermionic operators into account, which act on the half-link connected to $x$, i.e.,

$$
\hat{E}_{x, \mu}=1-\hat{n}_{x, \mu}^{a}-\hat{n}_{x, \mu}^{b}=\left[\hat{\eta}_{x, \mu}, \hat{\eta}_{x, \mu}^{\dagger}\right],
$$

which is valid in the link-symmetry invariant space $\left(\hat{L}_{x, \mu}-2\right)|\Phi\rangle=0$. As a consequence, in the Hilbert space with two Dirac fermions per link, the Gauss law generators become strictly local, i.e., containing quantum variables belonging solely to site $x$; they read

$$
\hat{G}_{x}=\hat{\psi}_{x}^{\dagger} \hat{\psi}_{x}-\frac{1-p_{x}}{2}-\sum_{\mu}\left(1-\hat{n}_{x, \mu}^{a}-\hat{n}_{x, \mu}^{b}\right) .
$$

Within this picture, it is easy to identify a local gaugeinvariant basis for the dressed site

$$
\begin{aligned}
\left|\begin{array}{ccc} 
& k_{4} & \\
k_{1} & \phi & k_{3} \\
& k_{2}
\end{array}\right|_{x} & =(-1)^{\delta_{k_{1}, 2}+\delta_{k_{2}, 2}} \\
& \times|\phi\rangle_{x}\left|k_{1}\right\rangle_{x,-\mu_{x}}\left|k_{2}\right\rangle_{x,-\mu_{y}}\left|k_{3}\right\rangle_{x, \mu_{x}}\left|k_{4}\right\rangle_{x, \mu_{y}},
\end{aligned}
$$

where $|\phi\rangle_{x}=\left(\hat{\psi}_{x}^{\dagger}\right)^{\phi}|0\rangle$ for $\phi \in\{0,1\}$ describes the matter content, while $k_{j} \in\{0,1,2\}$ selects a state, from those in Eq. (5), for each respective half-link. The factor $(-1)^{\delta_{k_{1}, 2}+\delta_{k_{2}, 2}}$ accommodates the sign in Eq. (7). In this language, the Gauss law, cast as Eq. (10), simplifies to

$$
\phi+\sum_{j=1}^{4} k_{j}=4+\frac{1-p_{x}}{2}
$$

which fixes the total number of fermions in each dressed site, specifically four in the even sites and five in the odd sites. Equation (12) actually reduces the Hilbert space dimension of each dressed site from 162 to 35, and we use these 35 states as a computational basis for tensor network algorithms.

A fundamental feature of this language is that, since the total number of fermions at each dressed site is conserved, their parity is conserved as well; thus, the gauge-invariant model will not exhibit any Jordan-Wigner strings (outside the dressed sites) in the computational basis. An operative way to show this property is to consider that the Hamiltonian term $\hat{\psi}_{x}^{\dagger} \hat{U}_{x, \mu} \hat{\psi}_{x+\mu}$ decomposes as the product of $\hat{\psi}_{x}^{\dagger} \hat{\eta}_{x, \mu}$ and $\hat{\eta}_{x+\mu,-\mu}^{\dagger} \hat{\psi}_{x+\mu}$ : Each of these two factors is local (acts on a single dressed site) and commutes with the 
algebra of other dressed sites. The same applies to the magnetic plaquette term. In conclusion, by working on the dressed-site computational basis, we can employ standard (spin-model-like) tensor network techniques, without the requirement of keeping track of fermionic parity at each site [65-71]. Notice that this construction can also be exploited to perform quantum computations of twodimensional LGTs.

\section{Tensor network for 2D lattice gauge simulations}

In order to numerically simulate the quantum system, we use a two-dimensional TTN state to represent the many-body wave function $[51,72,73]$. We work in the computational 35-dimensional local basis for each dressed site, defined in the previous section, which automatically encodes the Gauss law. Operators appearing in the Hamiltonian (1) can be cast in this basis, either as local operators or by acting on a pair or plaquette of neighboring dressed sites (see Appendix $\mathrm{C}$ for the explicit construction). The extra link symmetry $\hat{L}_{x, \mu}=$ 2 must be enforced at every pair of neighboring sites. We do so by introducing an energy penalty for all states violating the link constraints. This penalty term is included in the optimization by a driven penalty method-similar to an augmented Lagrangian methodwhich is described in more detail in Appendix E. Under all other aspects, the TTN algorithm employed here for finding the many-body ground state follows the prescriptions of Ref. [74].

In the numerical simulations, we fix the energy scale by setting the coupling strength $t=-1$. Furthermore, we work within a sector with a fixed total charge $\hat{Q}$, by using standard techniques for global symmetry conservation in TNs [74-76]. We thus characterize the ground-state properties as a function of the mass $m$, the electric coupling $g_{e}$, and the magnetic coupling $g_{m}$.

In order to exploit the best performances of our TTN algorithm, we run simulations on square lattices $L \times L$, with the linear length $L$ being a binary power; in particular, we consider $L=4,8$, and 16 , and vary the TN auxiliary dimension (or bond dimension) up to $\chi \sim 300$. Depending on $L$ and the physical parameters, we obtain a convergence precision between about $10^{-2}$ and $10^{-5}$, sufficient to characterize the ground-state properties.

\section{ZERO CHARGE DENSITY SECTOR}

In this section, we focus on the zero charge density sector $\rho \equiv\langle\hat{Q}\rangle / L^{2}=0$, where there is a balance between matter and antimatter, and we analyze the ground state of Hamiltonian (1) within this subspace. Unless otherwise stated, we consider periodic boundary conditions. We characterize the ground state of the Hamiltonian by looking at the energy density $\langle\hat{H}\rangle / L^{2}$ and the particle density $\langle\hat{n}\rangle=\left(1 / L^{2}\right) \sum_{x}\left\langle\hat{n}_{x}\right\rangle$, where $\hat{n}_{x}=\left(\delta_{x, e} \hat{\psi}_{x}^{\dagger} \hat{\psi}_{x}+\delta_{x, o} \hat{\psi}_{x} \hat{\psi}_{x}^{\dagger}\right)$ counts how many charges are in the system, both positive and negative, i.e., fermions in even sites plus holes in odd sites. We start our analysis by first focusing on the case in which the magnetic coupling has been set to zero, $g_{m}=0$. Before detailing the numerical results, some analytically solvable limit cases should be considered. For large positive values of the bare mass $m \gg t$, the fluctuations above the bare vacuum are highly suppressed; the system exhibits a unique behavior since there is no competition between the matter term and the electric-field term in the Hamiltonian. Indeed, to construct particle-antiparticle pairs, the matter energy and the electric-field energy both contribute to an overall increase of the ground-state energy. In order to explore more interesting phenomena, we allow the mass coupling to reach negative values. By doing so, we can identify two different regions, depending on the competition between the electric coupling $g_{e}^{2} / 2$ and the values of the mass $m<0$ :

(i) For $g_{e}^{2} / 2 \gg 2|m|$, we still have a vacuumlike behavior, where we expect a unique nondegenerate ground state with small particle-density fluctuations. This regime exists, no matter the value of the mass, as long as the energy cost to turn on a nonvanishing electric field on a single link overcomes the gain in creating the associated particle-antiparticle pairs. Indeed, for any value of the mass and $g_{e}^{2} / 2 \rightarrow \infty$, or for $g_{e}^{2} / 2 \neq 0$ and $m \rightarrow \infty$, the presence of a finite electric field, or finite particle density, is strictly forbidden, and the ground state flows toward the only admissible configuration, namely, the bare vacuum.

(ii) For $-2 m \gg g_{e}^{2} / 2>0$, the system is characterized by slightly deformed particle-antiparticle dimers; this regime of course only exists for negative values of the mass and represents the region wherein the energy gain for creating a particle-antiparticle couple largely overcomes the associated electric-field energy cost. Here, the ground state remains highly degenerate as long as the kinetic energy coupling $|t|$ is much smaller than all the other energy scales (with the degeneracy being lifted only at the fourth order in $t$ ). In particular, for $g_{e}^{2} / 2 \neq 0$ and $m \rightarrow-\infty$, the ground state reduces to a completely filled state. In order to minimize the electric-field energy, particles and antiparticles are arranged in $L^{2} / 2$ pairs (where we are assuming $L$ even), sharing a single electric flux in between. All of these configurations are energetically equivalent, and their degeneracy corresponds to the number of ways in which a finite quadratic lattice (with open or periodic boundary conditions) can be fully covered with given numbers of "horizontal" and "vertical" dimers. This number scales exponentially with the system size as $\exp \left(L^{2} C / \pi\right)$ for $L \rightarrow \infty$, with $C \simeq 0.915966$, the Catalan's constant [77]. For the sake of clarity, we stress that such dimers are not entangled 


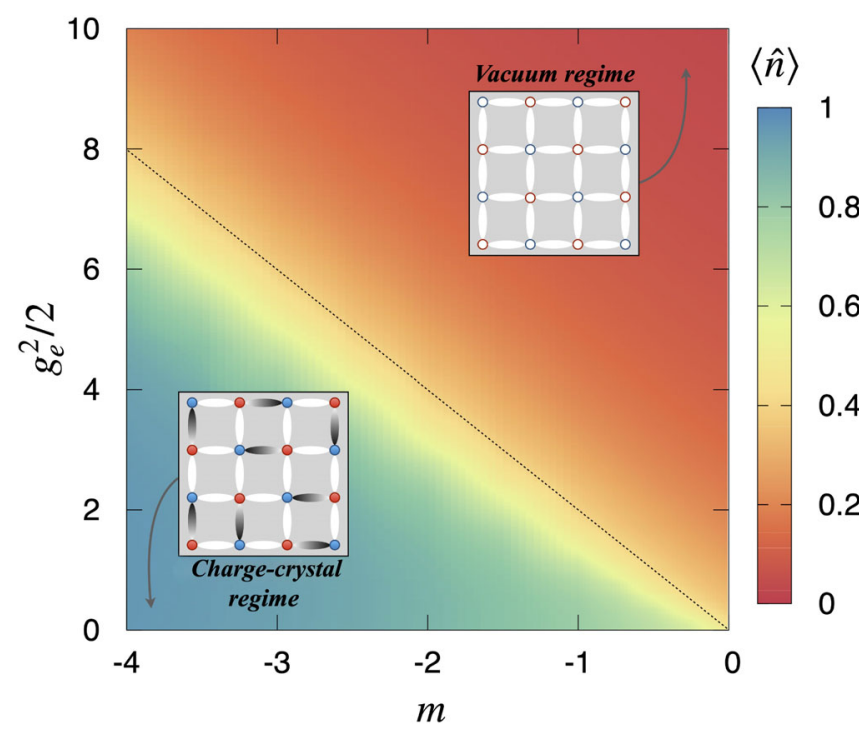

FIG. 3. Color density plot for $m<0$ obtained from the evaluation of the density of matter in the TTN ground state for an $8 \times 8$ lattice system with periodic boundary conditions. The insets are schematic representations of the ground state deep in the two regimes: the bare vacuum for $g_{e}^{2} / 2 \gg 2|m|$ and a typical dimer configuration for $g_{e}^{2} / 2 \ll-2 m$. The dashed line is located at the classical $(t=0)$ transition $g_{e}^{2} / 2=-2 m$.

clusters of matter and gauge fields; they are roughly product states.

Let us mention that the case $g_{e}=0$ with $m \rightarrow \infty$ $(m \rightarrow-\infty)$ is more pathological since any gauge-field configuration compatible with the vacuum (dimerized)

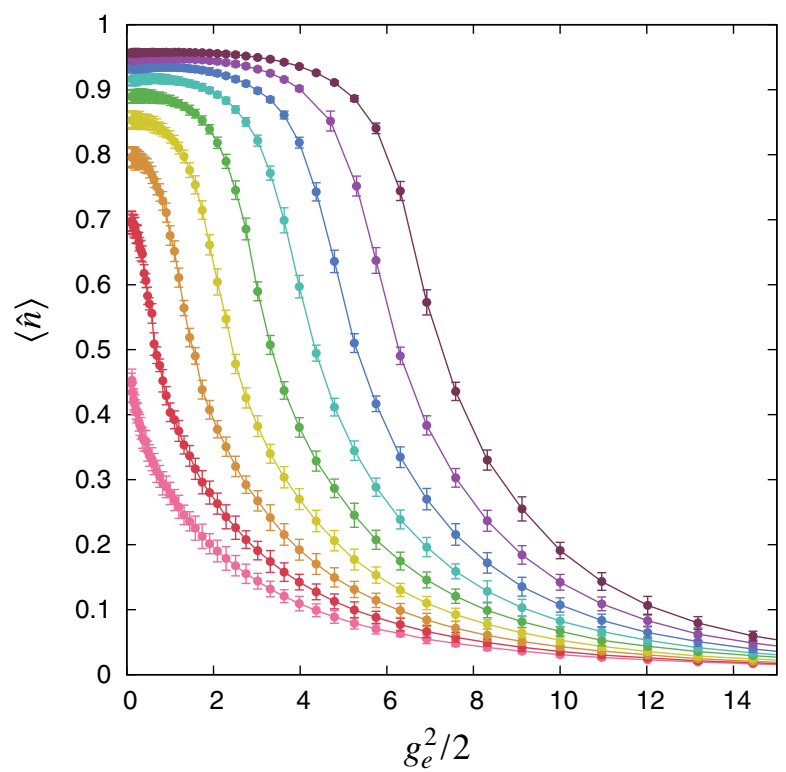

state is admissible, provided the Gauss law is fulfilled. In practice, we may draw a generic closed loop with finite electric flux on top of the vacuum state without modifying its energy; similar gauge loops may be realized on top of the dimerized state, provided it is compatible with the occupied links, without changing its energy. All of these configurations are gauge invariant by construction and increase the degeneracy of the ground-state energy sector.

Our numerical results confirm and extend this picture, as can easily be seen in the phase diagram displayed in Fig. 3, obtained from TTN simulations in an $8 \times 8$ system. The matter density is roughly zero in the vacuum regime; otherwise, it takes on a finite value whenever the system exhibits "dimerization", i.e., in the charge-crystal regime. We check that the numerical data, both the groundstate energy density and the particle density, show an asymptotic tendency toward the perturbative estimates. Interestingly, the particle density experiences an abrupt change mainly in a narrowed region around $m \simeq-g_{e}^{2} / 4$, where the local slope becomes steeper as the electric coupling (and the mass) approaches zero (see left panel in Fig. 4), as roughly predicted by perturbation theory and supported by the exact results in the $2 \times 2$ case (see Appendixes $\mathrm{H}$ and I).

The two regimes exhibit opposite long-range ordering, identified by the order parameter $\hat{\mathcal{O}}_{x}=(-1)^{x}\left(2 \psi_{x}^{\dagger} \psi_{x}-1\right)$, and are mutually frustrated in the proximity of the transition. As a confirmation of this property, we can track, for instance, the full density-density correlation function

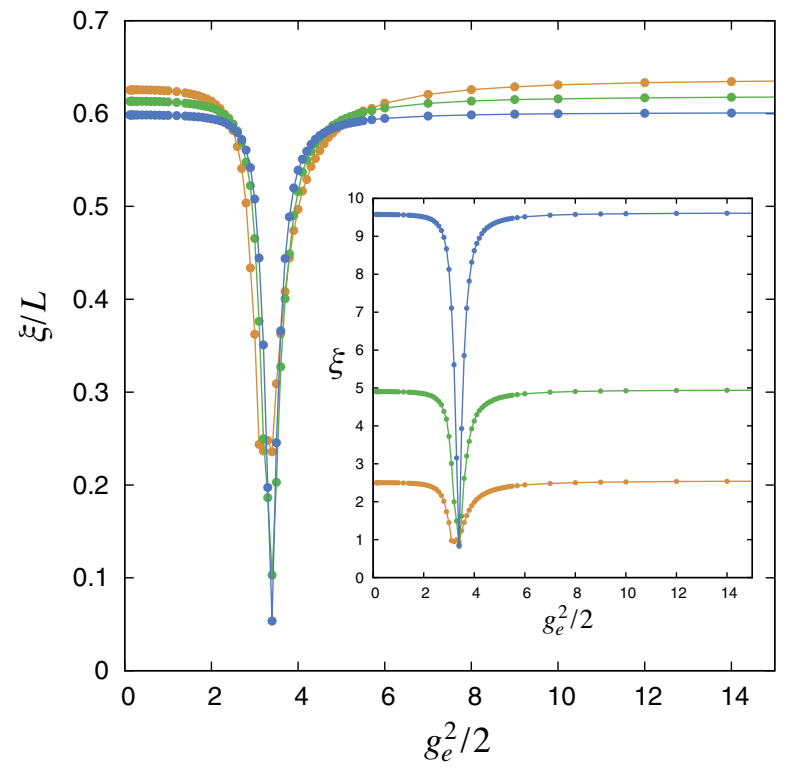

FIG. 4. Left panel: profiles of the matter density as a function of the electric-field coupling for different values of negative mass obtained by vertically cutting the color plot in Fig. 3. From bottom (pink circles) to top (purple circles), the mass takes the values $m \in\{-0.01,-0.5,-1,-1.5,-2,-2.5,-3,-3.5,-4\}$. Right panel: correlation length, in the ground state for $m=-2$ and varying the electric coupling, extracted from the density-density correlation function as explained in the main text. Different colors represent different sizes: $4 \times 4$ (orange), $8 \times 8$ (green), and $16 \times 16$ (blue). 
$\mathcal{C}_{x, x^{\prime}}=\left\langle\hat{\mathcal{O}}_{x} \hat{\mathcal{O}}_{x^{\prime}}\right\rangle$ (as compared to its connected component $\left.\mathcal{C}_{x, x^{\prime}}^{0}=\mathcal{C}_{x, x^{\prime}}-\left\langle\hat{\mathcal{O}}_{x}\right\rangle\left\langle\hat{\mathcal{O}}_{x^{\prime}}\right\rangle\right)$. We expect both regimes to exhibit an extensive (linear with $L$ ) full correlation length, while a sudden drop in such a quantity identifies frustration and helps us locate the transition with high precision. Such behavior is shown in the right panel of Fig. 4, where we quantify the full correlation length via the estimator $\xi_{\text {est }}^{2}=\sum_{v} D^{2}(v) \overline{\mathcal{C}}(v) / \sum_{v} \overline{\mathcal{C}}(v)$, which uses the spatially averaged correlation function $\overline{\mathcal{C}}(v)=L^{-2} \sum_{x} \mathcal{C}_{x, x+v}$ and the Euclidean metric $D^{2}(v)=v_{x}^{2}+v_{y}^{2}$. Such an estimator effectively calculates the two-dimensional variance of $\overline{\mathcal{C}}(v)$ meant as a distribution (further discussed in Appendix F). By testing sizes up to $16 \times 16$, we observe that the actual transition point is slightly below the classical (i.e., $t=0$ ) position $g_{e}^{2} / 2=-2 m$. Such an outcome confirms our predictions that particle-antiparticle fluctuations, induced by a finite value of the hopping amplitude $t$, naturally discourage the charge-crystal order.

This effect emerges already at the second order in the perturbation theory treatment (see Appendix $\mathrm{H}$ ), where the crossing point of the two different ground-state energies, $E_{v}$ (vacuum) and $E_{d}$ (dimer), slightly shifts toward the dimerized configuration.

A relevant physical question is whether the system undergoes an actual quantum phase transition across the two regions. Exactly at $t=0$, when $m$ crosses the critical value $-g_{e}^{2} / 4$, the ground state exhibits an exact level crossing, passing from the bare vacuum to the chargecrystal energy sector. In this limit case, the system experiences a trivial first-order phase transition since the gauge-field energy term and the matter-field mass term commute between each other. However, if we tune the mass at the classical critical value $m=-g_{e}^{2} / 4$, a small hopping amplitude $t \neq 0$ is already sufficient to remove such a degeneracy: Namely, the bare-vacuum energy and the charge-crystal energy get modified in a different way so that a gap opens between the two sectors. At the critical value of the mass, creation or annihilation of particleantiparticle pairs has no energetic cost, and the ground-state energy sector is characterized by all possible states with any number of dimers; however, creating a pair in the vicinity of the bare vacuum is more favorable than annihilating a pair on top of a fully dimerized state; this is at least true for any finite $L$ (see Appendix $\mathrm{H}$ for details).

A crucial insight comes from the features of the overlap between the exact ground state $|G S\rangle$ and the unique bare vacuum $|\Omega\rangle$ (see Appendix I for exact results in the $2 \times 2$ case). Indeed, for the $t=0$ trivial case, it experiences a discontinuous transition when passing from the vacuum sector to the full dimerized sector, suddenly jumping from one to zero. Interestingly, for fixed system size $L$, we may evaluate such an overlap in the approximate ground state $\left|G S^{(k)}\right\rangle$ at a given order $k$ in perturbation theory. The resulting perturbative expansion of the square of the overlap $\left|\left\langle\Omega \mid G S^{(k)}\right\rangle\right|^{2}$ changes continuously in the vacuum regime, while it remains identically vanishing, i.e., $\left|\left\langle\Omega \mid G S^{(k)}\right\rangle\right|^{2}=0$, when correcting the fully dimerized state up to $k<L^{2} / 2$. We thus expect the exact overlap to be continuous at the transition point and identically vanish in the thermodynamic limit $L \rightarrow \infty$. As a consequence, for any finite $t$, no first-order phase transition occurs, and we may have a second-order phase transition. Let us stress that, although the perturbative expansion of the fully dimerized state does not produce any change in the overlap with the bare vacuum for $k<L^{2} / 2$, local observables do experience perturbative modifications, simply because the state by itself gets modified. In particular, as a consequence of the Hellmann-Feynman theorem, the particle density as a function of the mass coupling $m$ coincides with the derivative of the ground-state energy density, $\langle G S(m)|\hat{n}| G S(m)\rangle=\partial_{m} E_{G S}(m) / L^{2}$. A secondorder phase transition will thus imply continuous profiles of the particle density, with a discontinuous or diverging derivative at the transition point. In fact, we have numerical evidence that the matter density changes continuously when going from one phase to the other (see Fig. 4); however, it remains very hard to infer its derivative at the transition point.

\section{A. Finite magnetic-coupling effects}

We now analyze the case of nonvanishing magnetic coupling $g_{m}$, especially focusing on how it impacts the many-body quantum features at zero temperature.

In Fig. 5, we show the field-plot representations of the ground-state typical configurations for an $8 \times 8$ system in the presence of magnetic couplings. For the sake of visibility, we only plot a $4 \times 4$ subsystem out of the complete $8 \times 8$ lattice simulated with periodic boundary conditions. Both mass $m$ and electric coupling $g_{e}$ have been chosen so that the system is deep within the two different regimes (left panels represent the vacuum regime; right panels represent the charge-crystal regime). As the magnetic coupling $g_{m}$ increases to commensurate values (bottom panels), we see negligible changes affecting the vacuum configuration. By contrast, in the charge-crystal regime, the nonvanishing magnetic coupling introduces a nontrivial reorganization of the electric fields.

Such an effect can be well understood in terms of perturbation theory: (i) In the vacuum region, the ground state is not degenerate, and the first nontrivial corrections are given by coupling such a state with all the states with a single flux loop over a single plaquette (whose energy is therefore $2 g_{e}^{2}$ ). In this regime, the flux loop state has high electric-field energy; thus, it will only slightly impact the global features of the state. The first-order correction to the ground-state energy will be quadratic in the magnetic coupling, i.e., proportional to $g_{m}^{4} / g_{e}^{2}$ (see Fig. 6, left panel). Let us stress that, even though the state may experience an 

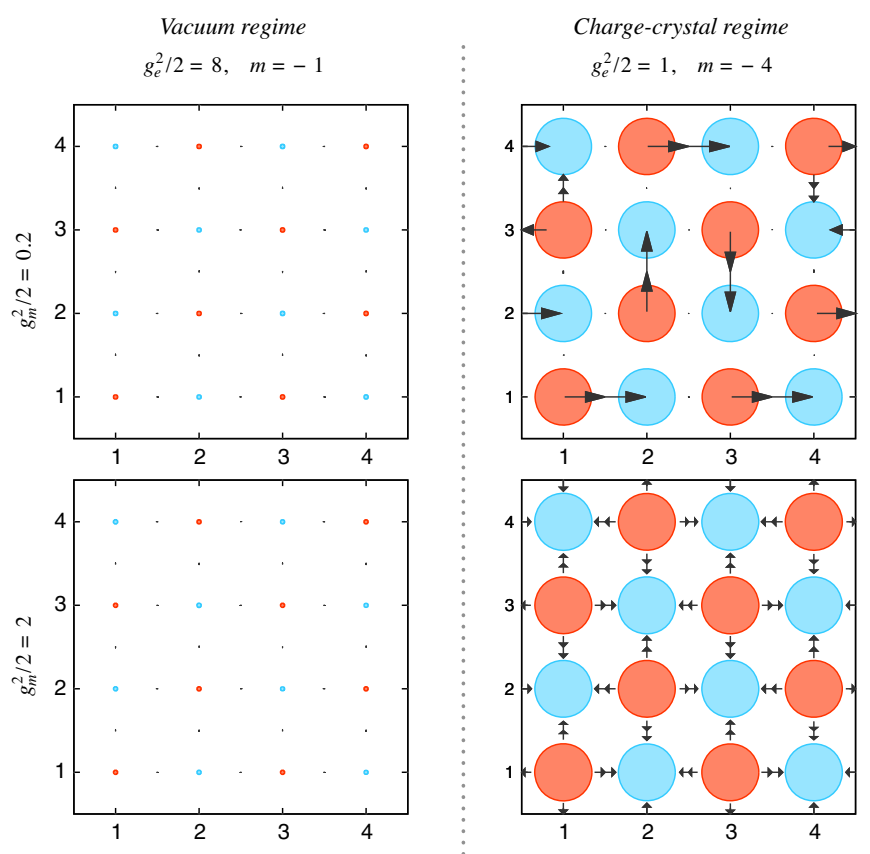

FIG. 5. Numerical results obtained via TTN simulations. The field plots reproduce the matter and gauge configurations for a $4 \times 4$ subsystem embedded in an $8 \times 8$ lattice with periodic boundary conditions. Red (blue) circles represent particles (antiparticles): Their diameter indicates the average density, from 0 (empty sites) to 1 (completely filled). The arrows in between represent the electric field: Larger arrows indicate greater electric flux.

electric-field reconfiguration due to the "field-loop" superpositions, the fact that in this regime the electric field is almost zero causes no visible effect on its expectation value; this is pretty clear from the left column of Fig. 5:
When passing from a small $\left(g_{m}^{2} / 2=0.2\right)$ to a slightly bigger value $\left(g_{m}^{2} / 2=2\right)$ of the magnetic coupling, the changes in the expectation value of the gauge field are negligible. (ii) In the charge-crystal configuration, the effect of the magnetic interaction is nontrivial. Indeed, the ground-state energy sector in this regime is highly degenerate, and the magnetic field contributes to lift such a degeneracy (at much lower order than $t$ ). The magnetic coupling introduces first-order transitions between different gauge-field configurations; therefore, its first contribution to the ground-state energy is scales like order $g_{m}^{2} / g_{e}^{2}$. Actually, a sufficiently large value of the magnetic coupling $g_{m}$ helps the TTN wave function to restore the square lattice symmetry by introducing a gap between the actual ground state and all the energetically unfavorable configurations. This property is noticeable in Fig. 5 (bottom-right panel), where for $g_{m}^{2} / 2=2$, the gaugefield distribution becomes uniform (on average) in the bulk. In this scenario, the charge crystal does not encourage the formation of dimers but instead a global entangled state of gauge fields.

The previous considerations are supported by the behavior of the ground-state energy and of the particle density, as a function of $g_{m}$. In Fig. 6, we plot the numerical results obtained via TTN simulations in an $8 \times 8$ system. We fix the value of the mass to $m=-2$ and explore the behavior for $g_{e}^{2} / 2=2$ and 6 , which are slightly below or above the classical transition point $g_{e}^{2} / 2=-2 m$. We vary the magnetic coupling in a rather big interval $g_{m}^{2} / 2 \in[0,8]$. In the first case, i.e., when the system is initially in the chargecrystal configuration, we expect linear corrections to the ground-state energy as a function of $g_{m}^{2}$; this is pretty clear from Fig. 6, where, however, some deviations are visible

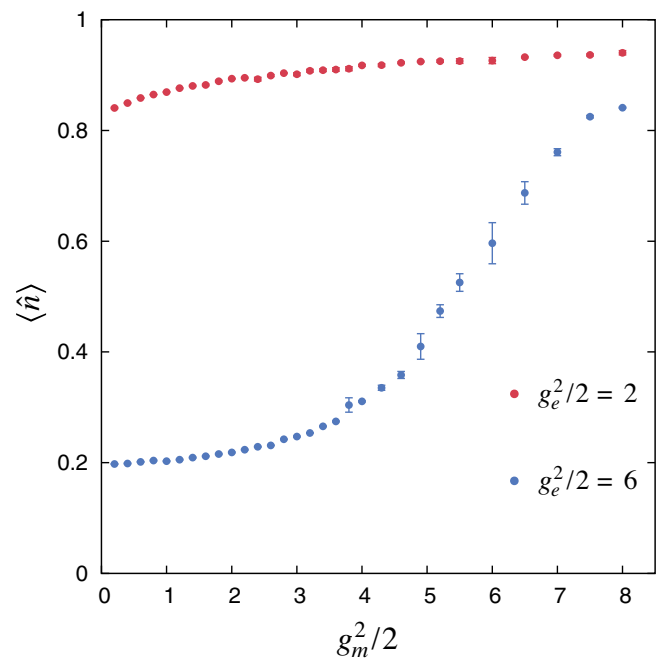

FIG. 6. Numerical results for the ground-state energy $\langle\hat{H}\rangle / L^{2}$ density and the particle density $\langle\hat{n}\rangle$ as a function of the magnetic coupling for an $8 \times 8$ system. The mass coupling has been fixed to $m=-2$. The energies have been renormalized to their absolute value at $g_{m}=0$; dashed lines are guides for the eye. Data have been obtained by extrapolating from TTN simulations with different auxiliary dimensions. 
because of the vicinity of the phase boundary. In the second case, i.e., starting from a quasivacuum, a quadratic deviation of the ground state is clearly visible (see Fig. 6).

Interestingly, in the parameter region we are exploring, the magnetic coupling enhances the production of particles, thus increasing the average matter density, even though the magnetic term does not directly couple to matter. Such an emergent behavior is physically relevant since it also arises when performing phase diagram simulations along a physical line of the QED problem. Specifically, setting $g_{e} g_{m}=8 t^{2}$, we realize the physical scenario of QED. Figure 11 in Appendix A shows a growing charge density at smaller QED couplings $g$, even when the (negative) bare mass is small.

In practice, the magnetic coupling creates resonating configurations of the gauge fields in the crystal charge regime, thus decreasing the electromagnetic energy density of the state itself, which in turn favors the crystal charge configuration in the proximity of the phase boundary. Hence, small $g_{m}$ values effectively enlarge the chargecrystal regime. However, in the spin-1 representation of the gauge field, the dimerized configuration is not stable under an arbitrary large value of the magnetic coupling, and we expect $\left\langle\hat{n}_{x}\right\rangle=0$ when $g_{m} \gg g_{e}$. This case can be easily understood at the classical level $(t=0)$, comparing the effect of a Wilson loop operator in the zero-matter (vacuum) sector and in the full-matter sector: In the former case, each single plaquette resonates between three different diagonal gauge-field configurations $\{|\circlearrowleft\rangle,|\varnothing\rangle,|\circlearrowright\rangle\}$; in the last case, only two configurations are resonating, e.g., $\{|\uparrow \downarrow\rangle,|\leftrightarrows\rangle\}$, since constructing a clockwise (anticlockwise) electric loop $\circlearrowright(\circlearrowleft)$ on top of the first (second) state is forbidden by the spin-1 finite representation. This leads to a contribution of the magnetic coupling to the energy, which is proportional to $-\sqrt{2} g_{m}^{2}$ for a plaquette in the vacuum, while it is only $-g_{m}^{2}$ for a dimerized plaquette. In practice, such a difference remains at the many-body level as well, and, therefore, although for $-2 m \gg g_{e}^{2} / 2$ the dimerized configuration represents the lower energy state at $g_{m}=0$, it will become energetically unfavorable for sufficiently strong magnetic couplings.

We have further analytical confirmation of such behavior from the exact diagonalization of $2 \times 2$ systems (see Appendix I for details): For a single plaquette system, the first visible effect of a nonvanishing magnetic coupling is to mix up the two dimerized states into two different superpositions with different energies. The transition between the vacuum state toward the lower energetic charge-crystal state is therefore sharpened, and its position is shifted as well in $g_{e}^{2} / 4+m \simeq\left(g_{e}^{2}+g_{m}^{2} / 2-\sqrt{g_{e}^{4}+g_{m}^{4} / 2}\right) / 4$. Interestingly, depending on the values of $g_{e}$, this shifting is not monotonous in $g_{m}$, producing an initial increase in the particle density followed by a definitive decrease toward zero (cf. Fig. 19) and thus confirming the previous heuristic argument based on perturbation theory. Again, this shifting is a strictly finite-spin representation effect, and it disappears as the spin gets larger, as shown by analyzing the behavior for the single plaquette in the spin-2 compact representation of the gauge field (see Appendix I).

\section{FINITE CHARGE DENSITY SECTOR}

One of the most intriguing phenomena we observed in our numerical simulations relies on the possibility to create a charge imbalance in the system. This scenario is challenging for Monte Carlo techniques as it produces the sign problem $[13,24]$. Instead, our gauge-invariant tensor network approach is very well suited to overcome such difficulty: The fact that the global $U(1)$ symmetry has been explicitly embedded in the tensor network ansatz [74] allows us to work exactly within each sector with fixed total charge. In the following, we only consider $g_{m}=0$. Moreover, in this setup, because of the finite net electric flux coming out of the entire system, we have to work with open boundary conditions. In this geometry, the dressed sites at the boundary are now characterized by one outgoing half-link (two in the corners), which can support the electric field to allow the existence of a nonvanishing total outgoing flux.

When a finite density of charge $\rho \equiv\langle\hat{Q}\rangle / L^{2} \in\{-1 / 2,1 / 2\}$ is injected into the system, we expect a different behavior, depending on which part of the phase diagram the ground state belongs in. Indeed, when the ground state is very close to the bare vacuum, any charge created on top of it is forced to reach the boundaries so as to minimize the total energy; this is easily understood already with the classical $(t=0)$ Hamiltonian, and there are no fluctuations of the gauge fields. In this case, a classical configuration with a single charge located at distance $\ell$ from the boundary costs at least $\ell g^{2} / 2$ more than the optimal configuration where the same charge is located at the surface (see Fig. 7). In this regime, the diagonal energy term gets modified as $E_{v} / L^{2}=\left(g_{e}^{2} / 4+m\right) \rho$, as long as $\langle\hat{Q}\rangle \leq 2(L-1)$, i.e., whenever the total excess of charge is lower than the number of allowed free sites at the boundaries. When the total charge increases, deeper sites start to be filled; e.g., for $2(L-1)<\langle\hat{Q}\rangle \leq 4(L-2)$, one starts filling the nextneighboring sites to the surface (e.g., Fig. 8). Overall, this argument supports the existence of a phase separation between a boundary region attached to the surface, or strip, where charges aggregate and a bulk region expelling charges and electric fields. In the picture where the gauge field is not truncated $(S \rightarrow \infty)$, both regions will scale as a surface. In practice, defining $\rho_{\ell} \equiv 2 \ell(L-\ell) / L^{2}$ as the maximum amount of charge density the system can store within a strip of extension $\ell$ from the surface, we have a sharp discontinuity in local charge densities, at the smallest $\ell^{*}$ such that $\rho \leq \rho_{\ell^{*}}$, between a finite-charge region (for $j<\ell^{*}$ ) and a zero-charge region (for $j>\ell^{*}$ ). In particular, in the thermodynamic limit, we obtain $\ell^{*} / L=(1-\sqrt{1-2|\rho|}) / 2$. In other words, the width $\ell^{*}$ of the surface strip where all charges are localized varies 


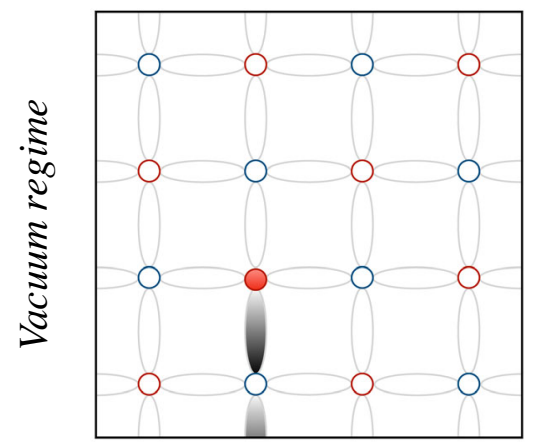

$\langle\hat{H}\rangle=m+3 g_{e}^{2} / 4$

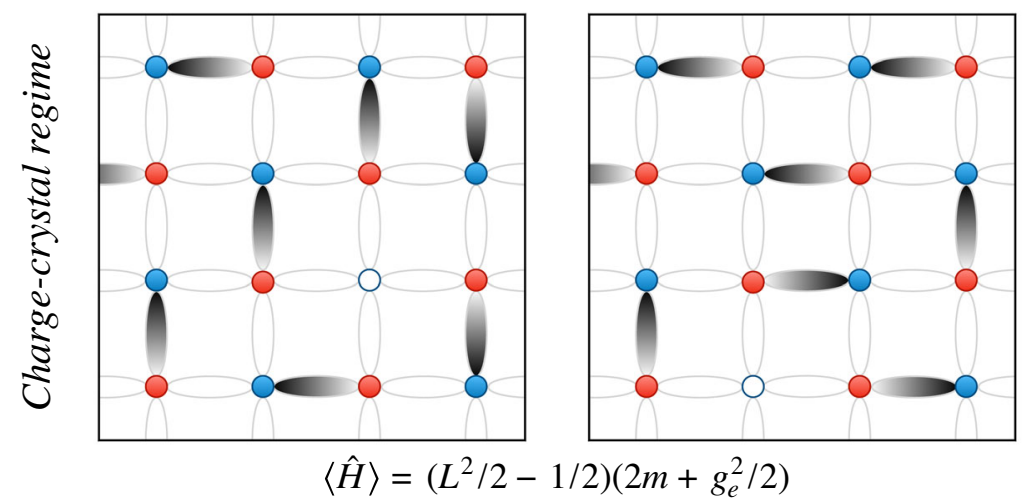

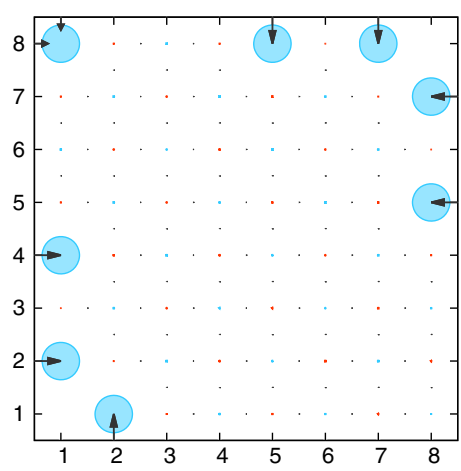

$\langle\hat{H}\rangle=m+g_{e}^{2} / 4$

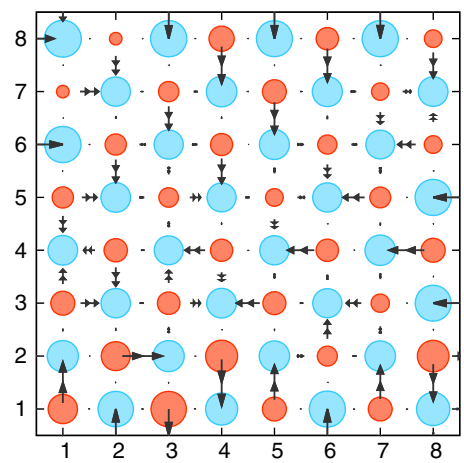

FIG. 7. Field plots in the finite charge density sectors. The top row refers to the vacuum regime and the bottom row to the chargecrystal regime. On the left of the figure, the four panels represent a sketch of the classical configurations (i.e., $t=0)$ for a $4 \times 4$ system with open boundary conditions in the $Q=1$ charge sector. Gauge fields can now exit the system at the energy cost of a half-link each the system at the cost of the half-link. The panels on the right of the figure are the field plots obtained by numerical TTN simulations in $8 \times 8$ systems for the two different regimes, namely, $g_{e}^{2} / 2=2$ and $m=4$ (top) or $m=-2$ (bottom), and charge sector $Q=-8$. In the vacuum regime, the excess of charge prefers to be localized at the boundaries since such configurations are more energetically favorable. In the dimerized regime, the holes may occupy any position since the system can reconfigure the pairs of dimers so as to always use the same amount of energy. However, because of the very high degeneracy of the low-energy sector, the TTN simulations may get stuck in a slightly asymmetric configuration.

smoothly in $[0, L / 2]$ as $|\rho|$ varies in $[0,1 / 2]$. Quantitatively, both the depth of the surface strip $\left(\ell^{*}\right)$ and the diameter of the bulk region $\left(L / 2-\ell^{*}\right)$ scale linearly with $L$; thus, the phase
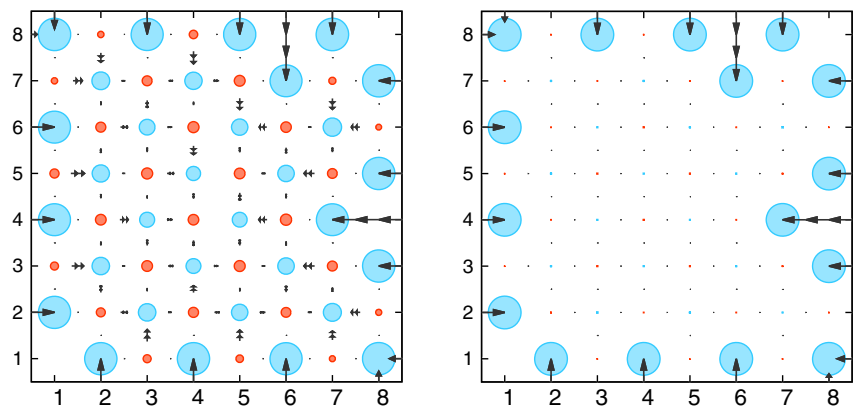

FIG. 8. Field plots from the TTN numerical simulations of $8 \times 8$ systems in the $Q=-16$ charge sector. The couplings are tuned in such a way that the system is deep in the vacuum regime (right panel, $g_{e}^{2} / 2=2, m=5$ ) or near the critical region (left panel, $\left.g_{e}^{2} / 2=2, m=-1\right)$. Notice how the excess of charge is larger than the allowed antimatter sites at the surface: The system has to allocate two extra charges in the next-neighboring sites to the surface. separation argument applies when approaching the thermodynamic limit, as long as the gauge field is unconstrained and the lattice spacing stays finite. In practice, as long as the average charge density is finite, we always have an extensive region in the bulk of the system whose linear dimension scales as $L \sqrt{1-2|\rho|} / 2$, which exhibits no charges. However, we stress that when introducing a fixed truncation of the gauge field (to any spin $S$ ), the amount of total charge that can be injected in the system is limited to a linear scaling in $L$ since, due to the Gauss law, the total electric flux at the boundary must match the total charge. Therefore, in order to approach the thermodynamical limit at finite charge density, one needs to increase the truncation or introduce static background fields.

We expect this picture to be slightly modified at finite hopping coupling $|t|$ but to remain valid as long as the system belongs to the vacuum regime. In practice, a finite tunneling amplitude introduces a small homogeneous particle density, thus slightly increasing $\ell^{*}$ and also building up a finite charge penetration length scaling linearly with $|t|$ such that the phase separation is 

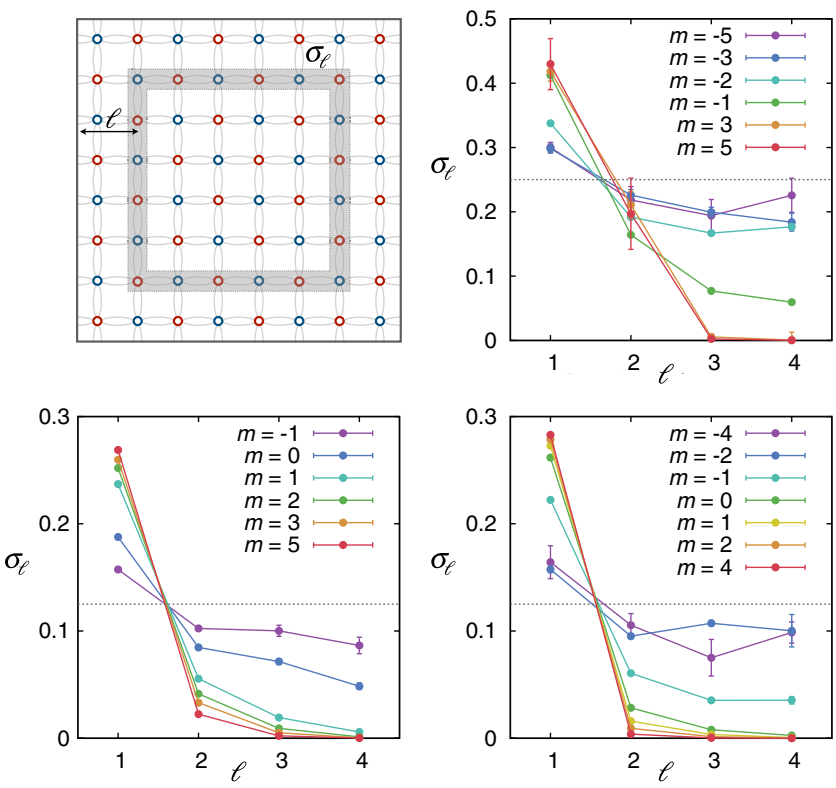

FIG. 9. Surface charge density evaluated in an $8 \times 8$ system as sketched in the top-left image. The shaded region represents the domain $\mathcal{D}_{\ell}$ defined in the main text. TTN simulations have been performed for different charge sectors and electric couplings; in clockwise order, $\left(Q=-16, g_{e}^{2} / 2=2\right),\left(Q=-8, g_{e}^{2} / 2=2\right)$, and $\left(Q=-8, g_{e}^{2} / 2=1 / 2\right)$.

smoothened out with an exponentially small density-charge tail penetrating into the bulk. The overall scenario is confirmed by the field plots in Figs. 7 and 8, where it is pretty clear that when the couplings are tuned in order for the system to be deep in the vacuum regime, the excess charges stick to the boundary so as to minimize the length of the attached electric strings. In principle, all possible configurations with all charges at the boundaries are energetically equivalent. However, the TTN many-body wave function spontaneously breaks such symmetry and picks up a single, specific configuration, as is usually the case with DMRG-like algorithms. We stress that such phase separation, where both bulk and boundary regions scale extensively, is likely an artifact of the lattice discretization, where the amount of local charge density is bound.

When the state belongs to the charge-crystal regime, a finite positive (negative) charge density is mainly generated by creating holes in the odd (even) sublattice; namely, negative (positive) charges are removed from the fully dimerized state. In order to minimize the energy, the holes can now be fully delocalized: A hole in the bulk, or at the boundary, generates a reconfiguration of the charge-crystal state in such a way that it always requires the same amount of energy and guarantees the expected total outgoing electric flux. In this regime, the zero-order energy term gets modified as $E_{d} / L^{2}=\left(g_{e}^{2} / 4+m\right)(1-\rho)$. The entire system is now characterized by a unique spatial phase where we expect a uniform average charge density and finite electric field in the bulk. Let us mention that, for any finite value of the hopping amplitude, we still expect a similar behavior, where the transition toward the phase-separated phase will be driven by the competition between the mass and the electric coupling.

In order to highlight the different features of the lowenergy state at finite chemical potential, we analyze the behavior of the surface charge density,

$$
\sigma_{\ell} \equiv \frac{1}{\operatorname{dim} \mathcal{D}_{\ell}} \sum_{x \in \mathcal{D}_{\ell}}\left\langle\hat{\psi}_{x}^{\dagger} \hat{\psi}_{x}\right\rangle
$$

where $\mathcal{D}_{\ell}$ is a square that counts $\operatorname{dim} \mathcal{D}_{\ell}=4(L+1-2 \ell)$ lattice sites as sketched in Fig. 9. Here, $\ell \in\{1,2, \ldots, L / 2\}$ represents the distance of the domain $\mathcal{D}_{\ell}$ from the external surface: Namely, as $\ell$ grows, we select domains deeper into the bulk.

In Fig. 9, we plot the surface charge density $\sigma_{\ell}$ as a function of $\ell$ for different points in the coupling-parameter space. As long as the Hamiltonian is tuned into the vacuum
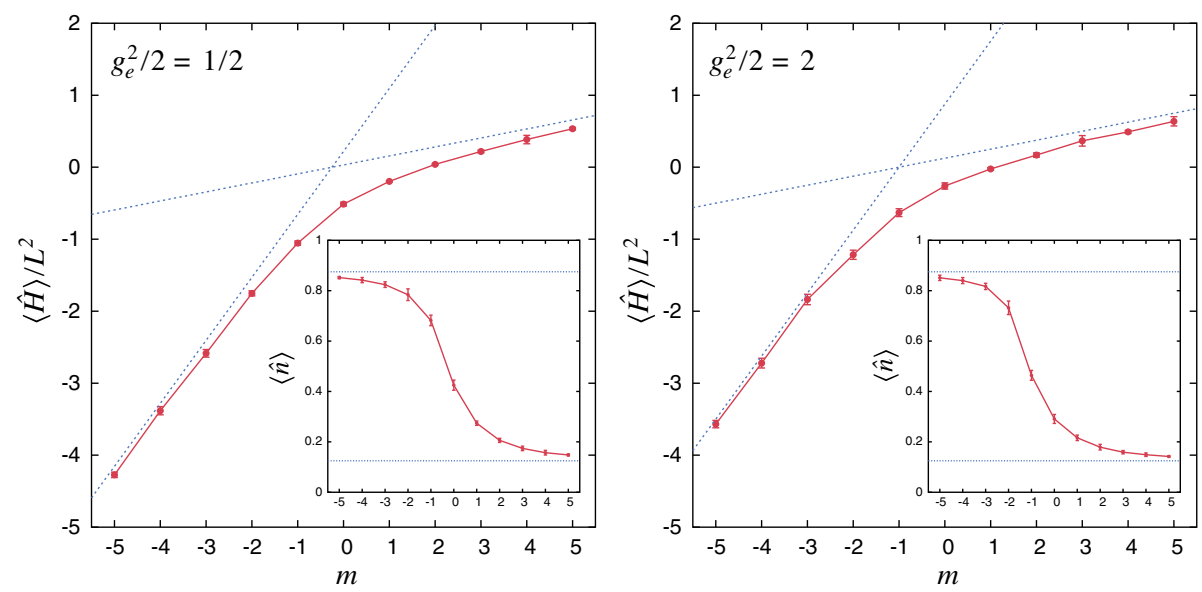

FIG. 10. Ground-state energy density and particle density (insets) as a function of the bare mass $m$ for an $8 \times 8$ lattice with $\rho=-1 / 8$, i.e., in the $Q=-8$ charge sector. In the left panel, the transition between the two regimes occurs at $m \sim-1 / 4$, while in the right panel, it is located at $m \sim-1$. Dashed lines are the asymptotic values in the zero-order perturbative approximation. 
regime, the surface charge suddenly drops when getting into the bulk of the system. As expected, for finite value of the couplings, when approaching the critical region, the bulk charge density is enhanced; finally, once the system reaches the charge-crystal regime, $\sigma_{\ell}$ acquires a loosely uniform shape.

Finally, we carefully check the ground-state energy density and the particle density, which are plotted in Fig. 10 as a function of the mass for two different values of the electric coupling. Notice how, for sufficiently large positive (negative) values of the mass, the data get closer to the perturbative predictions. The intermediate region, at $m \sim g_{e}^{2} / 4$, is characterized by stronger quantum fluctuations and thus exhibit a smooth transition between uniform and nonuniform charge distribution in space.

As a concluding remark, we stress that, while in this section we consider the boundary conditions to be completely free, setting a specific set of boundary conditions for the problem of electrodynamics is not conceptually or numerically difficult. Typical boundary conditions are realized by means of a boundary Hamiltonian $H_{b}$ to be added to the bulk Hamiltonian $H$ from Eq. (1) (see Appendix $\mathrm{G}$ for details).

\section{CONCLUSIONS}

In this work, we demonstrated a novel, efficient, tensor network approach to the study of two-dimensional lattice gauge theories. By exploiting the quantum-link formulation of LGT, the fermionic rishon representation of quantum links, and unconstrained tree tensor networks, we investigated the equilibrium properties of a two-dimensional lattice QED within its first compact spin representation. We present results for lattice size up to $16 \times 16$, whose Hilbert space dimension is approximately equivalent to that of a system composed of spins- $1 / 2$ on a square lattice with edges of about 80 lattice sites. Whenever possible, we confirmed our results with perturbative analysis and smallscale exact simulations.

In particular, we identified different regimes at zero chemical potential, a vacuum state and a charge-density one, that reproduce what has been found in the onedimensional case, and we investigated the effects of a magnetic term uniquely present in two dimensions. Finally, we explored the finite density scenario and individuated two distinct behaviors corresponding to the vacuum and charge-density configurations: In the former case, the excess charges accumulate on the boundaries. This configuration minimizes the electric energy density, and is analogous to how charges distribute in classical conductors. In the latter, the excess charge is distributed uniformly in the bulk and boundaries.

In conclusion, we have shown that unconstrained tree tensor networks are a powerful tool to obtain a nonperturbative description of a lattice gauge theory in two dimensions. We stress that these simulations have been obtained on standard clusters without exploiting heavy parallelization and with simulations lasting only a few days. Despite the fact that the presented results are not yet able to allow physical predictions in the continuous limit for the system we study, we foresee that upgrading the current software to exploit the full power of high performance computing - without major changes in the algorithmslarger system sizes, an additional dimension, the continuum and large- $S$ limit, and more complex Abelian and nonAbelian lattice gauge theories will be in the range of the approach presented here, as already shown for the onedimensional case [31,61]. Our proposed architecture is perfectly tailored to accommodate advanced strategies of diagnostics, including elaborate string order parameters capable of detecting deconfined phases and topological order [78-80].

\section{ACKNOWLEDGMENTS}

We are very grateful to M. Dalmonte, K. Jansen, L. Salasnich, U. J. Wiese, and P. Zoller for valuable comments. The authors acknowledge support from the BMBF and EU-Quantera via QTFLAG and QTHEP, the Quantum Flagship via PASQuanS, the INFN, the MIUR via the Italian PRIN2017, the DFG via the TWITTER project, the US Air Force Office of Scientific Research (AFOSR) via IOE Grant No. FA9550-19-1-7044 LASCEM, and the Austrian Research Promotion Agency (FFG) via QFTE project AutomatiQ.

\section{APPENDIX A: PHYSICAL QED SCENARIO}

Complementing the discussions in Sec. III A, we present two physical lines of phase diagram simulations of the QED problem with $g_{e} g_{m}=8 t^{2}$. Figure 11 shows a growing charge density at smaller QED couplings $g$, even when the (negative) bare mass is small.
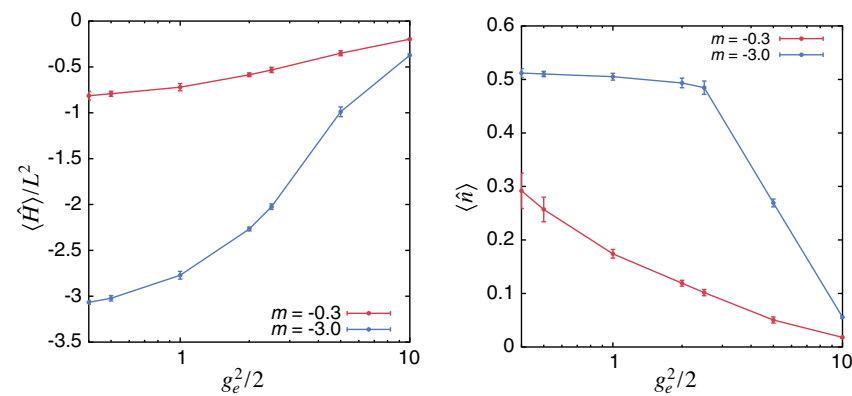

FIG. 11. Numerical results for the ground-state energy density $\langle\hat{H}\rangle / L^{2}$ and the particle density $\langle\hat{n}\rangle$ of the original QED Hamiltonian formulation as a function of the electric coupling $g_{e}$ for $8 \times 8$ systems. The lattice spacing is set to $a=1 / t=1$, and the magnetic coupling is tuned with respect to $g_{m}^{2}=8 /\left(g_{e}^{2} a^{2}\right)$. The mass coupling has been set to $m_{0}=\{-0.3,-3.0\}$, respectively. The data have been obtained by extrapolating from TTN simulations with different auxiliary dimensions. 


\section{APPENDIX B: CHARGE SCREENING}

Here, we briefly address the problem of detecting confinement. A natural way of exhibiting confinement in our lattice scenario is to show that electric-field lines do not extend over infinite lengths in the full-fledged theory (where $g_{e} g_{m}=2 \sqrt{2} t$ ), even when we enforce the presence of charges at specific locations. In this sense, the ground QED solution adjusts the mobile charges (and anticharges) to screen the pinned ones. We can insert such pinned charges by tuning a local chemical potential term $\tilde{m}_{x}(-1)^{x} \hat{\psi}_{x}^{\dagger} \hat{\psi}_{x}$, which shifts the mass at site $x$ to strongly negative values ( $\tilde{m}_{x}+m \ll-1$ ), thus favoring the presence of a full charge at $x$ in the ground state.

Adding a single pinned charge has the effect of creating a local excitation in the vacuumlike regime. Figure 12(a) shows a scenario with global zero charge, and a single pinned charge, under periodic boundaries. While the crystal-charge regime is mostly unaffected, in the vacuumlike regime, opposite-sign charges are attracted around the pinned one, so as to form an almost perfect meson, carrying an electric-charge quadrupole. Field lines propagating from this configuration are very short ranged, thus supporting confinement.

It is important to mention that confinement can be further corroborated by string-breaking analysis, where an initial (high-energy) configuration with a long field-line string breaks down to multiple localized mesons. Configurations with long (extensive) field lines can be engineered either by field linking a bulk charge to a point in the boundary, in the sector $Q_{\text {tot }}=1$, or by setting two pinned charges far apart

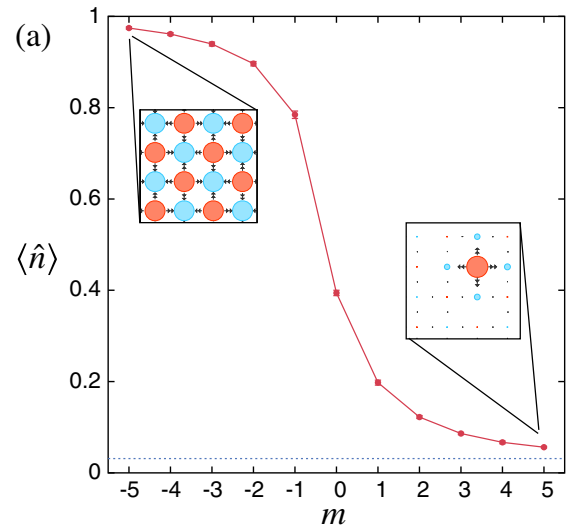

(b)

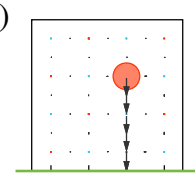

(c)

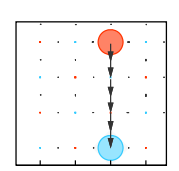

FIG. 12. Occupation for pinned charges in an $8 \times 8$ system of the original QED Hamiltonian with $g_{e}=1$ and $a=1 / t=1$. (a) Particle density for one charge pinned in the zero-charge sector $Q_{\text {tot }}=0$ with respect to the bare mass $m$ for periodic boundaries. The system transitions from the completely filled charge-crystal phase to the pinned charge screening. (b) Field plot for a system with total charge $Q_{\text {tot }}=1$ with open boundaries (green line). (c) Field plot for two pinned charges in the $Q_{\text {tot }}=0$ symmetry sector. All of the field plots are $4 \times 4$ subsystems embedded in an $8 \times 8$ simulation. For panels (b) and (c), the mass term is set to $m=4$.

in the $Q_{\text {tot }}=0$ sector. When the field lines (strings) scale in length with the system size, we expect them to be broken by the appearance of screening charges around the pinned ones, in the thermodynamical limit. By contrast, for finitelength strings, it is possible to set the bare mass sufficiently large so that they will remain unbroken, as shown in Figs. 12(b) and 12(c).

\section{APPENDIX C: CONSTRUCTING THE COMPUTATIONAL HAMILTONIAN}

In this section, we sketch the steps needed to obtain the operator matrices, and their elements, which appear in the computational formulation of the quantum-link QED model. In particular, we stress how to construct building-block operators $A_{j}^{(\alpha)}$, each acting on a single dressed site $j$, which are genuinely local, in the sense that they commute, by construction, with every other building-block operator at another site: $\left[A_{j}^{(\alpha)}, A_{j^{\prime} \neq j}^{\left(\alpha^{\prime}\right)}\right]=0$. The electric-field term and the bare mass term are diagonal in the occupation basis of fermions and rishons, as in Eq. (11), and thus trivially obtained. The nondiagonal terms are decomposed as follows:

Matter-field coupling terms.-Matter-field terms decompose naturally as $\psi_{x}^{\dagger} U_{x, x+\mu_{x}} \psi_{x+\mu_{x}}=A_{x}^{(1) \dagger} A_{x+\mu_{x}}^{(3)}$ (and its Hermitian conjugate) for horizontal "hopping" terms, and $\psi_{x}^{\dagger} U_{x, x+\mu_{y}} \psi_{x+\mu_{y}}=A_{x}^{(2) \dagger} A_{x+\mu_{y}}^{(4)}$ for vertical hopping. The decomposition into building blocks is based upon

$$
\begin{aligned}
\psi_{x}^{\dagger} U_{x, x+\mu_{x}} \psi_{x+\mu_{x}} & =\psi_{x}^{\dagger} \eta_{x, \mu_{x}} \eta_{x+\mu_{x},-\mu_{x}}^{\dagger} \psi_{x+\mu_{x}} \\
& =\left(\eta_{x, \mu_{x}}^{\dagger} \psi_{x}\right)^{\dagger}\left(\eta_{x+\mu_{x},-\mu_{x}}^{\dagger} \psi_{x+\mu_{x}}\right) \\
& =A_{x}^{(1) \dagger} A_{x+\mu_{x}}^{(3)},
\end{aligned}
$$

where $\eta_{x, \mu}$ are the three hardcore fermionic operators defined in Eq. (3). Both $A_{x}^{(1)}$ and $A_{x}^{(3)}$ are built on an even number of fermionic operators; therefore, they commute with any operator that does not act on site $x$ and are thus genuinely local. The vertical hopping term is similarly decomposed into building-block operators.

Magnetic terms.-The magnetic (or plaquette) term decomposes into building-block operators, acting on the four dressed sites at the corners of a plaquette. Specifically, we have

$$
\begin{aligned}
& U_{x, x+\mu_{x}} U_{x+\mu_{x}, x+\mu_{x}+\mu_{y}} U_{x+\mu_{y}, x+\mu_{x}+\mu_{y}}^{\dagger} U_{x, x+\mu_{y}}^{\dagger} \\
& \quad=\eta_{x, \mu_{x}} \eta_{x+\mu_{x},-\mu_{x}}^{\dagger} \eta_{x+\mu_{x}, \mu_{y}} \eta_{x+\mu_{x}+\mu_{y},-\mu_{y}}^{\dagger} \\
& \times\left(\eta_{x+\mu_{y}, \mu_{x}} \eta_{x+\mu_{x}+\mu_{y},-\mu_{x}}^{\dagger}\right)^{\dagger}\left(\eta_{x, \mu_{y}} \eta_{x+\mu_{y},-\mu_{y}}^{\dagger}\right)^{\dagger} \\
& \quad=-\left(\eta_{x, \mu_{y}}^{\dagger} \eta_{x, \mu_{x}}\right)\left(\eta_{x+\mu_{x},-\mu_{x}}^{\dagger} \eta_{x+\mu_{x}, \mu_{y}}\right) \\
& \times\left(\eta_{x+\mu_{x}+\mu_{y},-\mu_{y}}^{\dagger} \eta_{x+\mu_{x}+\mu_{y},-\mu_{x}}\right)\left(\eta_{x+\mu_{y}, \mu_{x}}^{\dagger} \eta_{x+\mu_{y},-\mu_{y}}\right) \\
& \quad \equiv-A_{x}^{(5)} A_{x+\mu_{x}}^{(6)} A_{x+\mu_{x}+\mu_{y}}^{(7)} A_{x+\mu_{y}}^{(8)},
\end{aligned}
$$


to be added, in the Hamiltonian, to its Hermitian conjugate. All operators in this decomposition are local and ready to use for TTN algorithms.

\section{APPENDIX D: TENSOR NETWORKS}

In what follows, we describe the background and main principles of tensor networks and, in particular, the TTN ansatz considered in this work. For a more in-depth description of TNs, we refer to more technical reviews and textbooks $[32,74,81,82]$.

TNs are used to efficiently represent (pure) quantum many-body wave functions $|\psi\rangle$, which live in the tensor product $\mathcal{H}=\mathcal{H}_{1} \otimes \mathcal{H}_{2} \otimes \cdots \mathcal{H}_{N}$ of $N$ local Hilbert spaces $\mathcal{H}_{k}$, each assumed to be of finite dimension $d$. Expressing such a state in the real-space product basis means decomposing the wave function as

$|\psi\rangle=\sum_{i_{1}, \ldots i_{L}=1}^{d} c_{i_{1}, \ldots, i_{L}}\left|i_{1}\right\rangle_{1} \otimes\left|i_{2}\right\rangle_{2} \otimes \ldots \otimes\left|i_{L}\right\rangle_{L}$,

where $\left\{|i\rangle_{k}\right\}_{i}$ is the canonical basis of site $k$, spanning $\mathcal{H}_{k}$. Describing such a general state by all possible combinations of local states requires $d^{N}$ coefficients $c_{i_{1}, \ldots, i_{N}}$. Thus, we have exponential growth with the system size $N$ in the exact representation of the wave function. For physical states, which satisfy certain entanglement bounds under real-space bipartitions (area laws) [83,84], tensor networks offer a more efficient representation. This representation is given by decomposing the complete rank- $N$ tensor into a set of local tensors with smaller rank, connected with auxiliary indices. We control the dimension of the auxiliary indices with the bond dimension $\chi$ and thereby the amount of captured information. Thus, tuning this parameter $\chi$, TNs interpolate between a product state, where quantum correlations are neglected, and the exact, but inefficient representation. The most prominent TN representations are the matrix product states (MPS) for 1D systems $[82,84,85]$ and their higher-dimension variant, the projected entangled pair states (PEPS) [83,86,87], while TTN [51,72,88,89] (as well as multi-scale entanglement renormalization Ansatz $[90,91])$ can, in principle, be defined in any lattice dimension.

Algorithms for MPS have been developed for over 20 years and have established the MPS ansatz as the primary workhorse for equilibrium problems in 1D [82,92] and, in many cases, even out of equilibrium [93,94]. By contrast, the development of TN algorithms, which are both quantitatively accurate and polynomially scalable, for twodimensional lattices is still ongoing. Currently, we are still facing the open question of which tensor network geometry is generally best suited for $2 \mathrm{D}$ simulations. The PEPS approximates the complete rank- $N$ tensor by a decomposition with one tensor for each physical site. These tensors are then connected through a grid analogous to the lattice, resulting in a TN with "loops" (nonlocal gauge redundancies). On the other hand, TTNs represent the wave function with a network geometry without loops, thus allowing (polynomially scaling) universal contraction schemes [74].

By its structure, the PEPS is the intuitive (and potentially more powerful) representation of a two-dimensional quantum many-body wave function satisfying the area laws of entanglement. However, in general, it lacks an exact calculation of expectation values. In fact, for a finite square lattice with $N=L \times L$ sites, the contraction of the complete PEPS to perform this calculation scales exponentially on an average system length $L$ [95]. Additionally, the optimization of the PEPS ansatz has a higher numerical complexity $\mathcal{O}\left(\chi^{10}\right)$ with the bond dimension, so the typical bond dimensions achieved are on the order of $\chi \sim 10$, which is sufficiently large for many spin systems with local dimension $d=2$. For the 2D LGT simulations presented in this work, however, we have to deal with a local dimension of $d=35$, which raises a nontrivial challenge for the PEPS ansatz. Furthermore, this local dimension increases for 3D systems or a higher representation for the discretization of the electric field.

The TTN, on the other hand, offers a more favorable computational scaling with bond dimension: Both exact full contraction and optimization algorithms scale with $\mathcal{O}\left(\chi^{4}\right)$, which in turn allows typical bond dimensions to even exceed $\chi \geq 1000$. Moreover, a TTN is fairly straightforward to implement and not restricted to any dimensionality of the underlying system; thus, the extension to 3D systems is straightforward. On the other hand, TTNs have been shown to poorly embed the area laws in two or higher dimensions [96]. Eventually, when increasing the system size, $N=L \times L$, the TTN may fail to accurately describe the quantum wave function. Thus, even though the TTN is a powerful tool to tackle systems in one, two, and three dimensions, further development and improvement is needed for reaching a scalable algorithm for higher system sizes. However, since this is a variational ansatz with increasing precision for increasing bond dimension, we can always give an estimate of the total error of our simulation results.

Our TTN algorithm implemented for finding the manybody ground state in this LGT analysis follows the prescriptions of Ref. [74]. In the numerical implementation, we exploit the $U(1)$ symmetry corresponding to the conservation of total charge $Q$ using common techniques for global symmetry conservation in TNs [76]. We construct the tree starting from the physical indices at the bottom by iteratively merging two local sites into one by a randomly initialized tensor coarse-grained site. In case we reach the maximum bond dimension for the coarse-grained space, we truncate the coupling symmetry sectors randomly in order to keep the bond dimension. Thereby, we randomly initialize not only the tensors themselves but the 


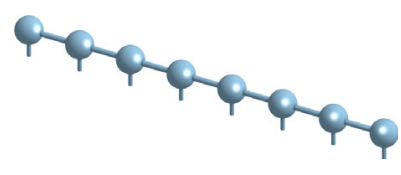

(a)

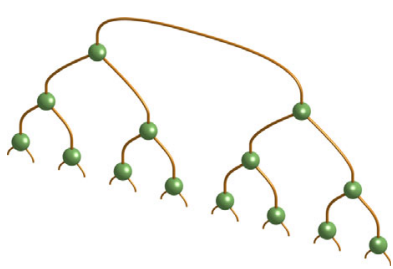

(c)

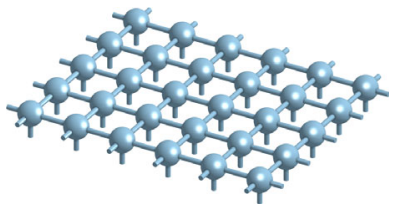

(b)

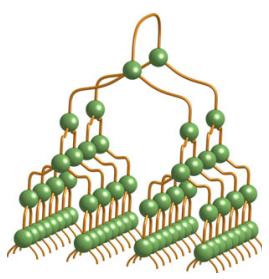

(d)
FIG. 13. Tensor network representations for a quantum manybody wave function: the matrix product states (MPS) (a) and the tree tensor network (TTN) (c) on the left hand side are for 1D systems, while the projected entangled pair states (PEPS) (b) and the TTN (d) on the right hand side represent 2D systems.

distribution of the coupling symmetry sectors within the tensors as well. In order to ensure convergence during the optimization, we dynamically increase the bond dimension locally, allowing us to adapt the symmetry sectors within the tree. In particular, we exploit the single tensor optimization with subspace expansion presented in Ref. [74], which approximates a two-site update by expanding the connecting link and iteratively optimizing the two local tensors separately. Thereby, we maintain the beneficial numerical complexity of $\mathcal{O}\left(\chi^{4}\right)$ instead of a heavier scaling of $\mathcal{O}\left(\chi^{6}\right)$ for the complete two-site optimization. For the single tensor optimization, we exploit the Arnoldi algorithm implemented in the ARPACK library. In this algorithm, the local eigenvalue problem is solved by iteratively diagonalizing the effective Hamiltonian $H_{\text {eff }}$ for the single tensor. It delivers the lowest eigenpairs of $H_{\text {eff }}$ up to a predefined precision $\epsilon$ by requiring only knowledge of the action of the operator $H_{\text {eff }}$. In the global optimization, we sweep through the TTN from the bottom to the top, performing the subspace expansion from each tensor towards its "parent" tensor [the one located directly above in the geometry of Fig. 13(d)]. After one complete sweep, we start over, iterating until global convergence (in terms of energy and selected observables) is achieved. As we come closer to convergence with each sweep, we also drive the optimization precision $\epsilon$ of the Arnoldi algorithm, such that we become more and more accurate in solving the local eigenvalue problems.

The computations with TTN presented in this work were run on different HPC clusters (the BwUniCluster and CINECA), where a single simulation of, e.g., an $8 \times 8$ system can last up to three weeks until final convergence, depending on the system parameters. Here, we point out that we can still improve the efficiency of the code and can have the potential to heavily parallelize our TTN to decrease the computational effort.

\section{APPENDIX E: TENSOR NETWORK SIMULATIONS FOR LATTICE GAUGE THEORIES}

In this section, we describe the TN approach for LGT in greater technical detail. As mentioned in Sec. II C, we already fulfill the Gauss law by choosing the local gaugeinvariant states (Sec. II B) as the logical basis in the TN simulations. In particular, we use an unconstrained TTN to represent the many-body wave function [51]. We adapt the TTN structure for the 2D system as shown in Refs. [72,73]. Following the description in Ref. [74], we additionally exploit the Abelian $U(1)$ symmetry, which corresponds to the total charge $\hat{Q}$ for the TTN representation. In this way, we keep the total charge $\hat{Q}$ fixed for each simulation by choosing the proper global symmetry sector.

As discussed in Sec. II A, the chosen local basis does not naturally respect the extra link symmetry arising from the division of the Hilbert space for each link into two halflinks. Thus, in additional to the LGT Hamiltonian $\hat{H}$ Eq. (1), we include a term to penalize the states violating the link constraint during the simulation. In conclusion, we simulate the Hamiltonian

$$
\hat{H}_{\text {sim }}=\hat{H}+\nu \sum_{x, \mu}\left(1-\delta_{2, \hat{L}_{x, \mu}}\right),
$$

with $\mu \in\left\{\mu_{x}, \mu_{y}\right\}$, where the penalty term vanishes when the link symmetry is respected and increases the energy for a state breaking the symmetry. Let us mention that this additional term translates to a nearest-neighbor interaction term in the TN simulations.

In theory, the penalty factor $\nu$ should be chosen as large as possible, as the link symmetry is strictly enforced for $\nu \rightarrow \infty$. But choosing a too-large $\nu$ leads to the optimization focusing on this penalty term only and fails to optimize for the physical quantities. Depending on the physical simulation parameter, $t, m, g_{e}$, and $g_{m}$, the penalty factor $\nu$ has to be chosen in a balanced way, such that we are able to optimize for the physical quantities as much as for the link constraint. In fact, when choosing $\nu$ too low, we end up with a result where the state does not strictly obey the link symmetry. If $\nu$ is too large, artifacts can appear in the proposed ground state, as the penalty term can introduce local minima and thus freeze the state in the optimization. These artifacts can either be a matter-antimatter pair for the vacuum regime or, as shown on the left in Fig. 14, a matterantimatter hole for the charge-crystal regime. As the total charge $\hat{Q}$ is strictly conserved by the chosen symmetry sector during the simulation, there are only two ways to get rid of such an artifact. The optimizer has to either locally violate the link symmetry or change the state at the neighboring sites together with the artifact—both of which would increase the energy in the simulation, given a large value for $\nu$. 

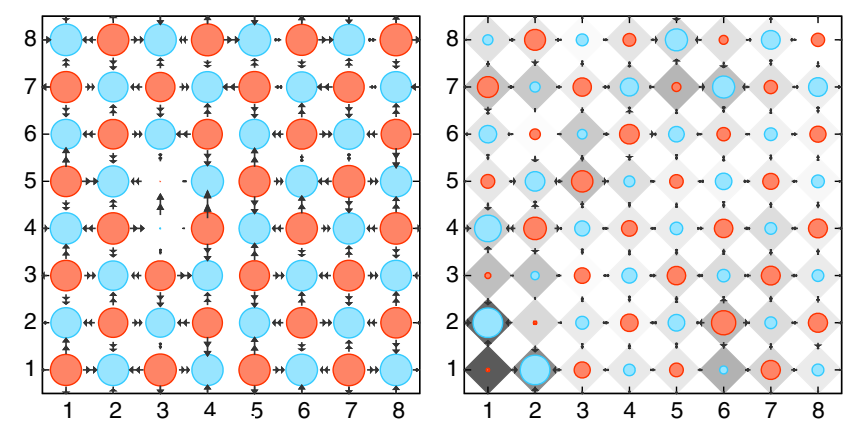

FIG. 14. Field plots from a TTN numerical simulation of $8 \times 8$ systems. The left panel depicts a configuration corresponding to a local minimum in the total energy in which a simulation got stuck because of a poor choice of the penalty parameter $\nu$. The right panel shows the field plot for a typical randomly initialized state. Note that in this case, the link symmetry is not respected. The gray diamonds in the background of each site signal the violation of this constraint. The darkness of the gray color corresponds to the contribution of the penalty term in Eq. (E1) for the site positioned at $x \equiv(i, j)$.

In order to improve this approach, we exploit two different methods. First, we start with a random state, which, in general, violates the link symmetry. One example for this random initialization is reported on the right side of Fig. 14. Second, we drive the penalty term by increasing $\nu$ after every optimization sweep. In particular, we start by linearly increasing $\nu$, until we observe an increment in the energy, which signals that the penalty term becomes significant for the optimization. Consequently, we switch to a quadratic tuning of $\nu$ such that, in the following few iterations, we increase $\nu$ more slowly than in the linear regime. Finally, we also set a maximum value for $\nu$, at which we stay for the rest of the optimization. The three different regimes of driving the penalty parameter $\nu$ are depicted in Fig. 15, showing the energy difference $\delta e$ to a higher bond dimension, together with $\nu$ with respect to the iterations for an exemplifying simulation.

With this driving, we optimize the random initial state in the first phase without focusing too strictly on obeying the link symmetry. This method flattens the local minima arising from including the penalty in the Hamiltonian and thereby helps converge to the global minimum. When choosing the linear tuning correctly, most physical observables are qualitatively already captured at the end of the first driving phase without strictly obeying the link symmetry. Thus, the second phase enforces the link symmetry, while the last phase-with a constant $\nu$ optimizes the state for the final quantitative ground state.

Although introducing the driven penalty drastically decreases the number of simulations that are stuck in artificial configurations, this cannot be completely avoided. Therefore, we simulate several samples with different random initial states. From these samples, we perform a

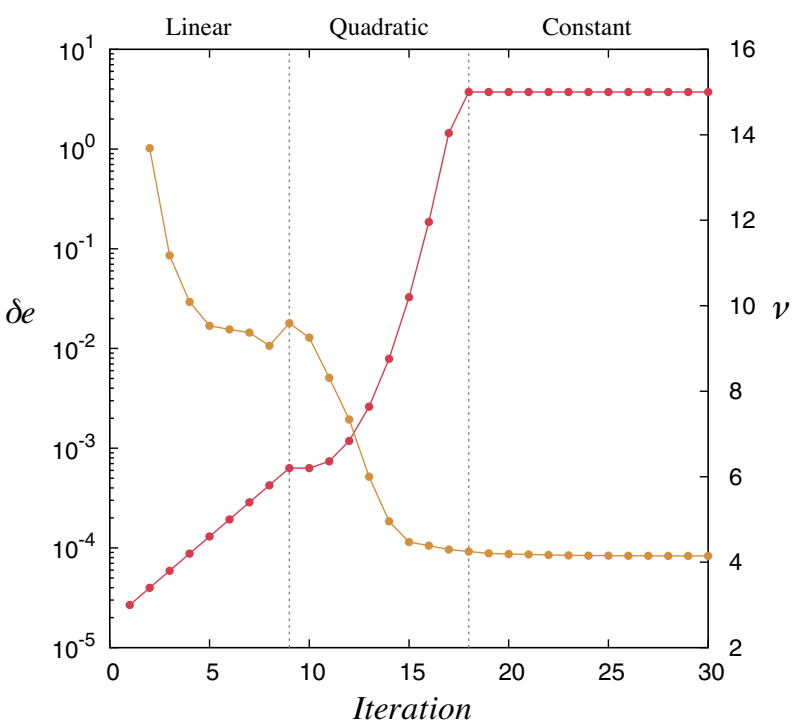

FIG. 15. Penalty parameter $\nu$ (red) and energy (yellow) with respect to the number of iterations for a typical LGT simulation. The energy is plotted here as a deviation $\delta e$ from the ground-state energy obtained with highest bond dimension available. We start with linearly increasing $\nu$. When the energy increases, we change to a quadratic driving regime with zero gradient at the transition point. Finally, we reach a predefined maximum value for $\nu$.

postselection and check whether the obtained wave functions are indeed physically correct ground states. We also observe the typical convergence for TN with increasing bond dimension when we discard the results with artifacts. From the different samples and the convergence in bond dimension, we can estimate the relative error in the energy, which, depending on the physical parameters, typically lies in the range of about $10^{-2}-10^{-4}$ for an $8 \times 8$ system.

\section{APPENDIX F: INTEGRAL ESTIMATORS FOR THE CORRELATION LENGTH}

Here, we briefly discuss a strategy to estimate correlation lengths based on integrals of the correlation functions. The obvious advantage is that this strategy employs all of the data within the correlation function itself while requiring no data regression. Therefore, it can be easily automatized and needs no careful initialization of the fit parameters for data regression; at the same time, it is a reliable, only slightly biased, estimator for correlation lengths [97].

While in the main text we applied an analogous estimator to the full correlation function, in this section, we perform an estimator analysis on the connected component $\mathcal{C}_{x, x^{\prime}}^{0}=\left\langle\hat{\mathcal{O}}_{x} \hat{\mathcal{O}}_{x^{\prime}}\right\rangle-\left\langle\hat{\mathcal{O}}_{x}\right\rangle\left\langle\hat{\mathcal{O}}_{x^{\prime}}\right\rangle$ of the correlation function, which we spatially average to $\bar{C}_{v}^{0}=L^{-2} \sum_{x} C_{x, x+v}^{0}$. In the absence of strong quantum correlations, lattice systems at 
low temperatures typically exhibit $\bar{C}^{0}(v) \simeq \alpha_{0} \exp (-|v| / \xi)$ exponentially decaying in the relative coordinate modulus $|v|=\sqrt{v_{x}^{2}+v_{y}^{2}}$, where $\xi$ is the actual correlation length and $\alpha_{0}$ is a (not interesting) prefactor. Here, we construct an integral estimator $\xi_{\text {est }}$ for (connected) correlation lengths and show that, on the exponentially decaying class, it returns $\xi$ to an acceptable precision.

In deriving these expressions, we assume that the system is much larger than the correlation length $L \gg \xi$ to avoid observing finite-size or boundary effects (we effectively approximate the lattice to $\mathbb{Z}^{2}$ ). For a $2 \mathrm{D}$ square lattice, we consider the following estimator:

$$
\xi_{\text {est }}^{2}=\frac{\sum_{v_{x}, v_{y} \in \mathbb{Z}}|v|^{2} \bar{C}^{0}(v)}{6 \sum_{v_{x}, v_{y} \in \mathbb{Z}} \bar{C}^{0}(v)}
$$

which, apart from the $1 / 6$ prefactor, is the (Euclidean) variance of $P(v)=\bar{C}^{0}(v)\left[\sum_{\mathbf{v}^{\prime}} \bar{C}^{0}\left(v^{\prime}\right)\right]^{-1}$, the correlation function normalized to a probability distribution over $\mathbb{Z}^{2}$ [assuming $\bar{C}^{0}(v)$ is symmetric, $\bar{C}^{0}(v)=\bar{C}^{0}(-v)$ ].

In the limit of correlation lengths that are large compared to the lattice spacing, $\xi \gg 1$, the discrete sums in Eq. (F2) converge to Riemann integrals,

$$
\xi_{\text {est }}^{2}=\frac{\int_{\mathbb{R}^{2}}|v|^{2} \bar{C}^{0}(v) d^{2} v}{6 \int_{\mathbb{R}^{2}} \bar{C}^{0}(v) d^{2} v},
$$

yielding an unbiased estimator $\xi_{\text {est }}^{2}=\xi^{2}$ for the family of correlation functions $\bar{C}^{0}(v) \simeq \alpha_{0} \exp (|v| / \xi)$. For correlation lengths comparable in magnitude to the lattice spacing, the finite sum in Eq. (F2) can produce a bias
$B(\xi)=\xi^{2}-\xi_{\text {est }}^{2} \geq 0$ in the estimator. Unfortunately, $B(\xi)$ is not an analytic function and thus cannot be removed altogether. However, we numerically verified that $B(\xi)$ is upper bounded by $1 / 17$, for any $\xi \in \mathbb{R}$, which makes Eq. (F2) a satisfactory estimator for the purposes of identifying phases and transitions.

\section{APPENDIX G: BOUNDARY HAMILTONIAN AND TYPICAL BOUNDARY CONDITIONS}

In this section, we discuss strategies to realize a specific set of (open) boundary conditions for problems of equilibrium electrodynamics. These strategies present an extension to the simulations realized in this work, which assume the boundary conditions to be either free (for finite charge density) or periodic (for zero charge density).

Von Neumann boundary conditions.-In this simple scenario, the outgoing electric flux at each boundary site is fixed and defined by the user. To realize this boundary condition, start the TTN algorithm from a product state that has the desired configuration of electric fluxes at the open boundary, and then simply carry out the optimization algorithm (without a boundary Hamiltonian, i.e., $H_{b}=0$ ). The algorithm has no means of changing the electric fluxes at the boundaries and will converge to the bulk ground state, given that specific boundary flux configuration.

Dirichelet boundary conditions.-To model the scenario where the boundaries are a perfect conductor, we actually assume the boundaries to be superconductive and expel magnetic fields by displaying huge magnetic couplings at the boundary. This model requires the usage of a magnetic boundary Hamiltonian

$$
\begin{aligned}
H_{b}= & J_{b}\left(\sum _ { j = 1 } ^ { L - 1 } \left(\hat{U}_{(1, j),-\mu_{x}}^{\dagger} \hat{U}_{(1, j), \mu_{y}} \hat{U}_{(1, j+1),-\mu_{x}}+\hat{U}_{(j, L), \mu_{y}}^{\dagger} \hat{U}_{(j, L), \mu_{x}} \hat{U}_{(j+1, L), \mu_{y}}+\hat{U}_{(L, j+1), \mu_{x}}^{\dagger} \hat{U}_{(L, j), \mu_{y}}^{\dagger} \hat{U}_{(L, j), \mu_{x}}\right.\right. \\
& \left.+\hat{U}_{(j+1,1),-\mu_{y}}^{\dagger} \hat{U}_{(j, 1), \mu_{x}}^{\dagger} \hat{U}_{(j, 1),-\mu_{y}}\right)+U_{(1, L),-\mu_{x}}^{\dagger} \hat{U}_{(1, L), \mu_{y}}+U_{(L, L), \mu_{y}}^{\dagger} \hat{U}_{(L, L), \mu_{x}}+U_{(L, 1), \mu_{x}} \hat{U}_{(L, 1),-\mu_{y}}^{\dagger} \\
& \left.+U_{(1,1),-\mu_{y}}^{\dagger} \hat{U}_{(1,1),-\mu_{x}}+\text { H.c. }\right),
\end{aligned}
$$

which contains both edge terms (top rows) and corner terms (bottom rows). To address the problem of electrodynamics, the ground-state algorithm is carried out while setting $J_{b} \gg \max \left\{|t|,|m|, g_{e}^{2}, g_{m}^{2}\right\}$, ensuring that the magnetic fields will approach a constant value (equal to zero) at the boundary, once converged.

\section{APPENDIX H: PERTURBATION THEORY}

Here, we describe the corrections to the ground state in both regimes outlined in Sec. III. Let us start by considering particle fluctuations due to the presence of a small tunneling $|t|$. The system has periodic boundary conditions.

Perturbation around the vacuum state.-For $m \gg|t|$, the vacuum state (with zero energy) is corrected by strictly local particle-antiparticle fluctuations. The first nontrivial contribution comes from a local dimer excitation as depicted in Fig. 16, whose average energy is $2 m+g_{e}^{2} / 2$. The truncated Hamiltonian reads (apart from the sign of the tunneling coupling, which, however, does not affect the results) 


$$
H_{v}=\left[\begin{array}{c|ccc}
0 & t & \cdots & t \\
\hline t & 2 m+g_{e}^{2} / 2 & & \\
\vdots & & \ddots & \\
t & & & 2 m+g_{e}^{2} / 2
\end{array}\right],
$$

which is a $\left(1+2 L^{2}\right) \times\left(1+2 L^{2}\right)$ matrix. The correction to the vacuum energy is therefore

$$
E_{v}=\left[\frac{g_{e}^{2}}{4}+m-\sqrt{\left(\frac{g_{e}^{2}}{4}+m\right)^{2}+2 L^{2} t^{2}}\right] .
$$

Perturbation around the dimer state.-Small-order tunneling perturbations on top of the fully dimerized states are not sufficient to remove their degeneracy. The ground-state energy sector remains degenerate up to the fourth order in perturbation theory. Here, we focus on the smallest order energy corrections for one specific dimerized configuration and consider the possible excitations as depicted in Fig. 16. We now have two different excitation sectors, depending on where we remove a particle-antiparticle pair: When the pair is annihilated on top of a dimer, the energy cost is $2 m+g_{e}^{2} / 2$; otherwise, when we remove a pair in between two dimers, it costs $2 m-g_{e}^{2} / 2$. The number of possible configurations of the first type coincides with the number of dimers, i.e., $L^{2} / 2$; in the other case, we have $3 L^{2} / 2$ different possibilities. The full truncated Hamiltonian is still a $\left(1+2 L^{2}\right) \times\left(1+2 L^{2}\right)$ matrix, which now reads [apart from the overall extensive constant $\left.E_{N} \equiv\left(2 m+g_{e}^{2} / 2\right) L^{2} / 2\right]$
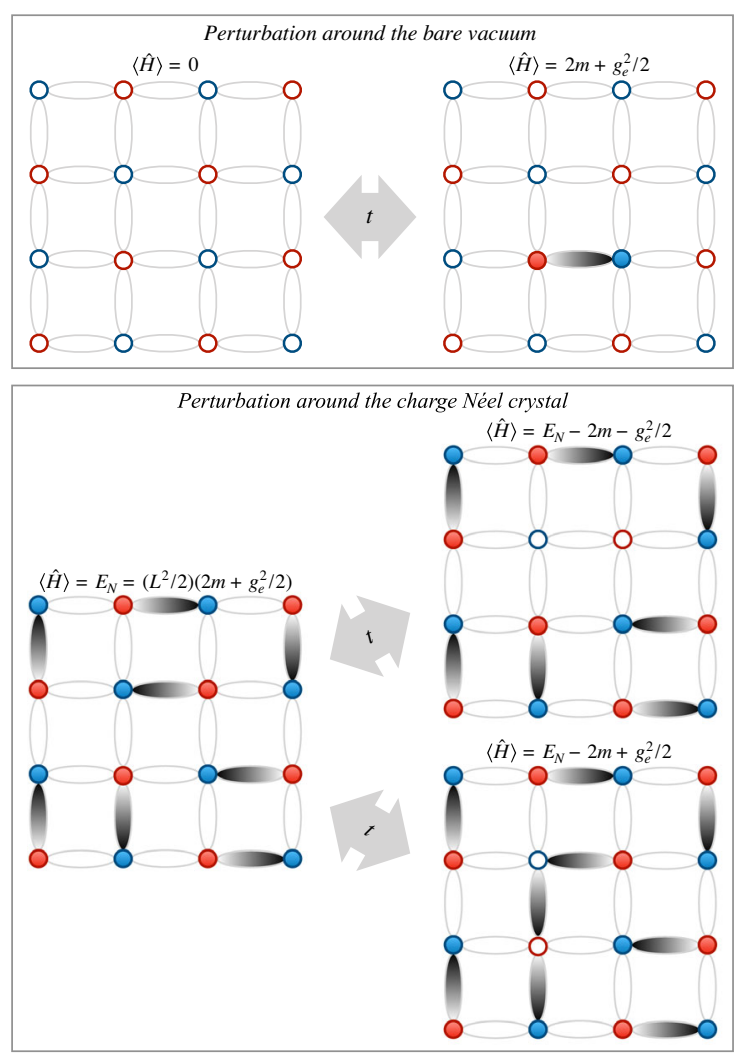

FIG. 16. Example of excited states coupled to the vacuum (top) or to the fully dimerized state (bottom) at the lowest order in perturbation theory in the tunneling coupling $t$, as described in Appendix H.

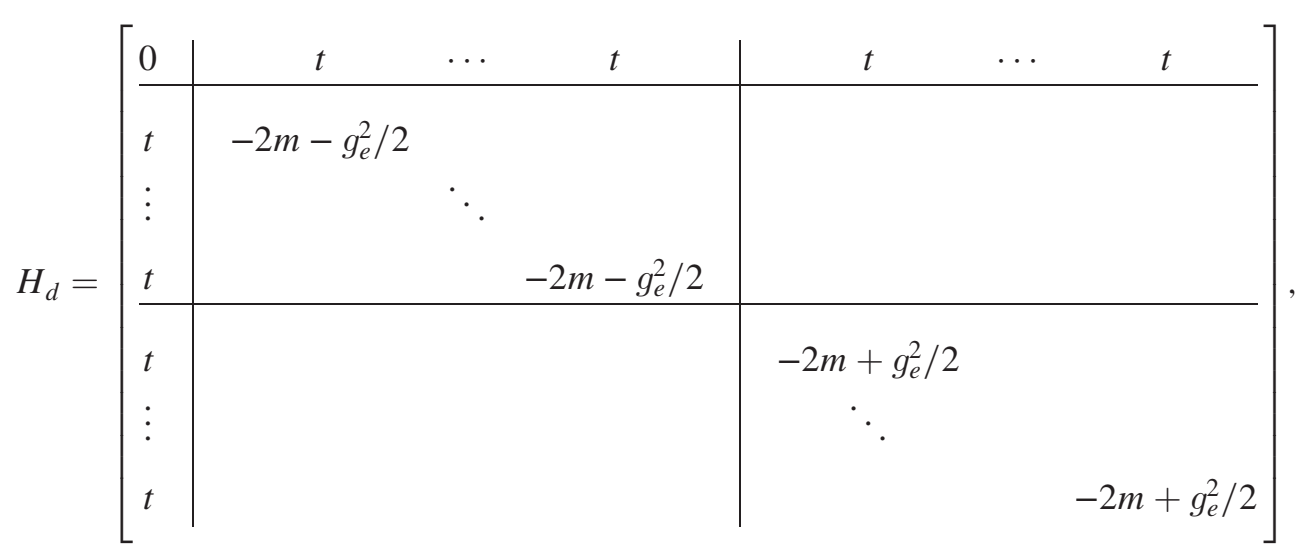

where, also in this case, the sign of $t$ does not affect the results. The correction to the vacuum energy can be evaluated as well by solving $\operatorname{det}\left(H_{d}-\varepsilon\right)=0$; indeed, because of the structure of the matrix, and thanks to the properties of the determinant, we find

$$
E_{d}=\frac{L^{2}}{2}\left(\frac{g_{e}^{2}}{2}+2 m\right)+\varepsilon_{-}
$$

where $\varepsilon_{-}$is the negative solution of

$$
\varepsilon\left[\frac{g_{e}^{4}}{4}-(2 m+\varepsilon)^{2}\right]+2 L^{2} t^{2}\left(\frac{g_{e}^{2}}{4}+2 m+\varepsilon\right)=0 .
$$

Now, it is clear that, when $m$ is approaching the value $-g_{e}^{2} / 4$, the biggest corrections, at the lower order in $t$, solely come from the sector that is quasidegenerate with the 

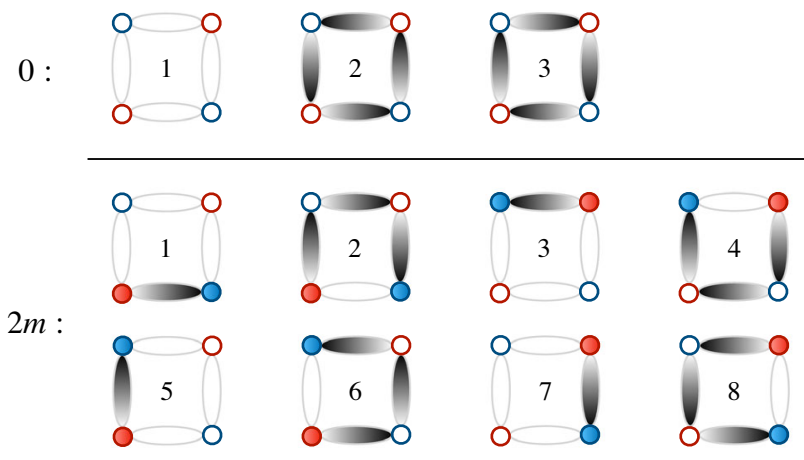

$4 m:$

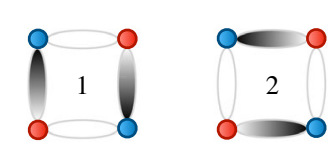

FIG. 17. Graphic representation of the basis vectors, in each mass sector, used to build up the $2 \times 2$ LGT Hamiltonian in the $S=1$ representation, as outlined in Appendix I.

classical dimerized configuration. The finite-size scaling depends on whether the mass is approaching from above (i.e., from the vacuum) or form below (i.e., from the dimerized configuration): In the first case, $2 L^{2}$ states contribute to the energy corrections; in the second case, if $g_{e}>0$, only $L^{2} / 2$ states get involved. Here, an energy gap, $\left|E_{v}-E_{d}\right| \sim L t / \sqrt{2}$, opens. Notice that, in the pathological situation where $g_{e}=0$ as well, there is no gap opening at the second order in $t$, and therefore a sharper transition is expected.

Let us mention that the correction to the groundstate energy coincides, as it should, with the secondorder degenerate perturbation theory. In practice, if $\hat{\mathcal{Q}}$ is the projector into the classical charge-crystal sector and $\hat{\mathcal{P}}=1-\hat{\mathcal{Q}}$ the projector into the complementary sector, then we may split the eigenvectors into two contributions: $\left|E_{k}\right\rangle=\left|\phi_{k}\right\rangle+\left|\varphi_{k}\right\rangle$, where $\left|\phi_{k}\right\rangle \equiv \hat{\mathcal{Q}}\left|E_{k}\right\rangle$ and $\left|\varphi_{k}\right\rangle \equiv \hat{\mathcal{P}}\left|E_{k}\right\rangle$. The eigenvalue equation $\left(\hat{H}_{0}-t \hat{V}\right)\left|E_{k}\right\rangle=$ $E_{k}\left|E_{k}\right\rangle$ therefore splits into two coupled equations:

$$
\begin{gathered}
-t \hat{\mathcal{P}} \hat{V}\left|\phi_{k}\right\rangle=\left(E_{k}-\hat{H}_{0}+t \hat{\mathcal{P}} \hat{V} \hat{\mathcal{P}}\right)\left|\varphi_{k}\right\rangle, \\
-t \hat{\mathcal{Q}} \hat{V}\left|\varphi_{k}\right\rangle=\left(E_{k}-E_{N}\right)\left|\phi_{k}\right\rangle,
\end{gathered}
$$

where we used the fact that, in our case, $\hat{\mathcal{Q}} \hat{V} \hat{\mathcal{Q}}=0$. Corrections within the degenerate subsector are thus given by recursively solving the following equation:

$\left(E_{k}-E_{N}\right)\left|\phi_{k}\right\rangle=\hat{\mathcal{Q}} \hat{V} \hat{\mathcal{P}} \frac{t^{2}}{E_{k}-\hat{H}_{0}+t \hat{\mathcal{P}} \hat{V} \hat{\mathcal{P}}} \hat{\mathcal{P}} \hat{V}\left|\phi_{k}\right\rangle$.

At the second order in the tunneling, the dimerized subsector degeneracy is not lifted, and the energy changes according to Eq. (H5). Let us stress that, deep in the charge-crystal regime, these are the dominant corrections. However, close to the classical transition, the creation or annihilation of a particle-antiparticle is energetically favorable, and nontrivial corrections to the degeneracy of the ground-state energy sector are induced by fourth-order tunneling transitions: Two different classical dimerized states are coupled whenever they share at least one "resonating" plaquette, which consists in two neighboring horizontal or vertical dimers (see the $4 \mathrm{~m}$ mass sector in Fig. 17). This effect partially removes the ground-state degeneracy, making a specific superposition of different dimer states energetically favorable. Incidentally, let us mention that, in the thermodynamic limit, there exist classical dimer configurations, e.g., the state where dimers are all vertically (horizontally) aligned with all local electric fluxes pointing in the same direction, which are not resonating with any other fully dimerized state at any order in perturbation theory.

\section{APPENDIX I: EXACT RESULTS OF THE $2 \times 2$ SYSTEM}

In the zero charge density sector, the single plaquette system, i.e., $2 \times 2$, admits only 13 gauge-invariant diagonal configurations in the spin-1 compact representation of the electric field. The full Hamiltonian can be easily constructed by considering each mass sector $\{0,2 m, 4 m\}$ independently, and it acquires the following block structure,

$$
H_{2 \times 2}=\left[\begin{array}{c|c|c}
D_{0} & T_{02} & \varnothing \\
\hline T_{20} & D_{2} & T_{24} \\
\hline \varnothing & T_{42} & D_{4}
\end{array}\right],
$$

where $D_{j}=D_{j}^{\dagger}, T_{20}=T_{02}^{\dagger}, T_{42}=T_{24}^{\dagger}$, and all matrix entries are real. To construct each block, we use the gauge-invariant eigenstates of the electric field $\hat{E}_{x, \mu}$ and particle number $\hat{n}_{x}$, as listed in Fig. 17.

The diagonal blocks read

$$
\begin{gathered}
D_{0}=\left(\begin{array}{ccc}
0 & -g_{m}^{2} / 2 & -g_{m}^{2} / 2 \\
-g_{m}^{2} / 2 & 2 g_{e}^{2} & 0 \\
-g_{m}^{2} / 2 & 0 & 2 g_{e}^{2}
\end{array}\right), \\
D_{2}=\mathbb{I}_{4} \otimes\left(\begin{array}{cc}
2 m+g_{e}^{2} / 2 & -g_{m}^{2} / 2 \\
-g_{m}^{2} / 2 & 2 m+3 g_{e}^{2} / 2
\end{array}\right), \\
D_{4}=\left(\begin{array}{cc}
4 m+g_{e}^{2} & -g_{m}^{2} / 2 \\
-g_{m}^{2} / 2 & 4 m+g_{e}^{2}
\end{array}\right),
\end{gathered}
$$

where $\mathbb{I}_{4}$ is a $4 \times 4$ identity matrix. The out-diagonal blocks are responsible for creation or annihilation of particleantiparticle pairs and are given by 

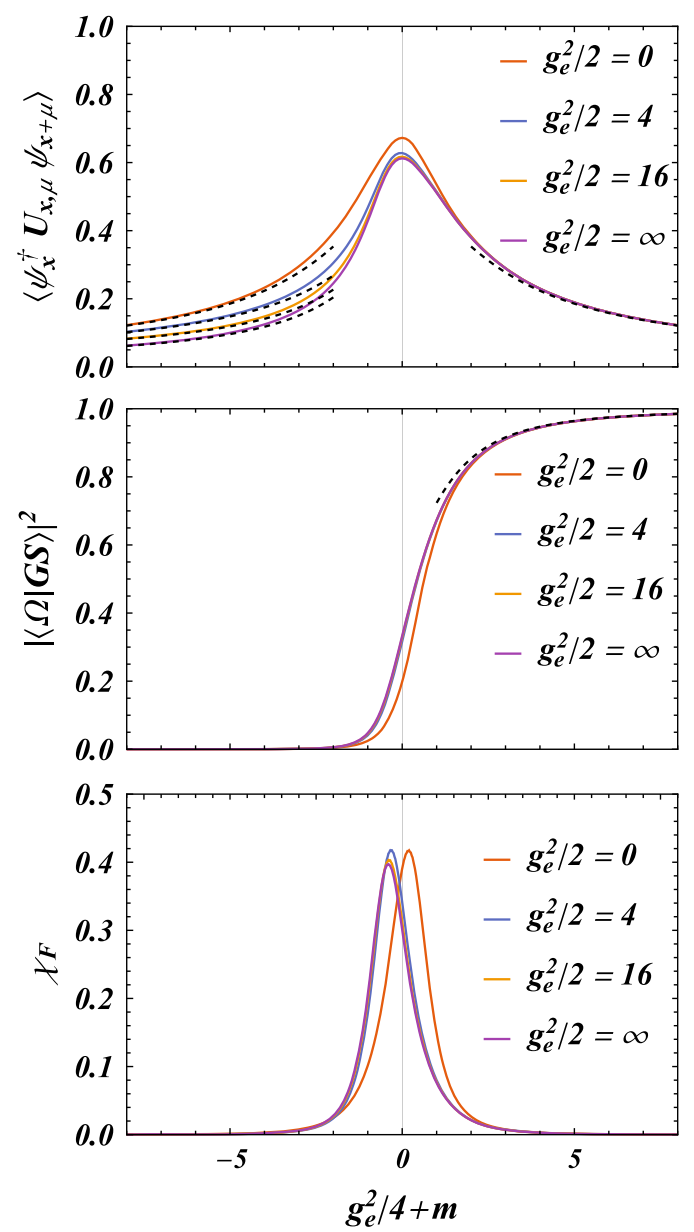

FIG. 18. Top panel: expectation value of the tunneling Hamiltonian as a function of the distance from the classical transition for a $2 \times 2$ system. Center panel: module square of the overlap between the exact $2 \times 2$ ground state and the vacuum state when varying the coupling across the classical transition point. Dashed lines are the perturbative predictions. Bottom panel: fidelity susceptibility of the ground state as defined in the main text.

$$
\begin{gathered}
T_{02}=\left(\begin{array}{cccccccc}
-t & 0 & -t & 0 & t & 0 & -t & 0 \\
0 & 0 & 0 & 0 & 0 & t & 0 & -t \\
0 & -t & 0 & -t & 0 & 0 & 0 & 0
\end{array}\right), \\
T_{42}=\left(\begin{array}{cccccccc}
0 & -t & 0 & -t & -t & 0 & -t & 0 \\
-t & 0 & -t & 0 & 0 & -t & 0 & -t
\end{array}\right) .
\end{gathered}
$$

The exact diagonalization of the Hamiltonian $\hat{H}_{2 \times 2}$ allows us to explore the behavior of the ground state in the vicinity of the transition $m \simeq g_{e}^{2} / 4$. As expected from the enhancement of quantum fluctuations, the gaugeinvariant hopping term gets picked at the transition (Fig. 18, top panel). The overlap of the ground state with the bare vacuum as a function of $m$ for different values of the electric coupling is analyzed as well (central panel in Fig. 18). Exact curves are compared with first-order perturbative results.

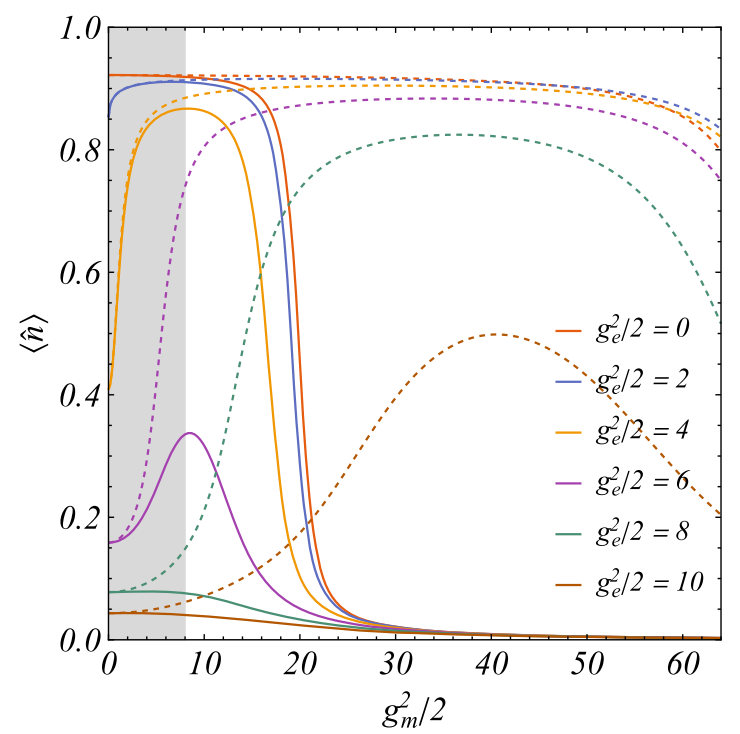

FIG. 19. Behavior of the particle density vs the magnetic coupling in the $2 \times 2$ system for $m=-2$ and different electric couplings $g_{e}^{2} / 2$. The shaded gray area represents the region explored in Fig. 6. Solid lines are the $S=1$ results; dashed lines are the $S=2$ results.

In order to more carefully explore the transition region, we look at the fidelity susceptibility of the ground state [98-101], $\chi_{F}(m) \equiv\left\langle\partial_{m} G S(m) \mid \partial_{m} G S(m)\right\rangle-\left|\left\langle G S(m) \mid \partial_{m} G S(m)\right\rangle\right|^{2}$, which gives the leading contribution to the ground-state fidelity $|\langle G S(m) \mid G S(m+\delta)\rangle|=1-\delta^{2} \chi_{F}(m) / 2+o\left(\delta^{2}\right)$, since the linear contribution in $\delta$ vanishes due to the normalization condition $\langle G S(m) \mid G S(m)\rangle=1$. This quantity is the perfect indicator of a change in the geometrical properties of the ground state when varying the couplings. Moreover, from perturbation theory, it can be easily shown that $\chi_{F}(m) \leq$ $\left[\left\langle G S(m)\left|\left(\sum_{x} \hat{n}_{x}\right)^{2}\right| G S(m)\right\rangle-\left\langle G S(m)\left|\sum_{x} \hat{n}_{x}\right| G S(m)\right\rangle^{2}\right] / \Delta^{2}$, where $\Delta$ is the energy gap between the ground state and the lower excitations. In practice, the fidelity susceptibility of the ground state is bounded from above by the number of particle fluctuations (which is an extensive quantity) divided by the gap. Whenever $\chi_{F}(m)$ shows a superextensive behavior, the ground state of the system should be gapless. From the numerical data, we have confirmation that $\chi_{F}(m)$ is enhanced in the vicinity of the transition between the two regions, as depicted in the bottom panel of Fig. 18.

In Fig. 19, we reproduce the behavior of the matter density as a function of the magnetic coupling, for different values of the electric-field couplings. As explained in the main text and confirmed by these exact results in the $2 \times 2$ plaquette, the local density gets enhanced by applying a small magnetic coupling; however, when $g_{m}^{2} \simeq g_{e}^{2}$, the particle density starts decreasing and eventually vanishes for $g_{m}^{2} \gg g_{e}^{2}$. Let us stress that this phenomenon is strictly due to the finite compact representation of the gauge field.

Indeed, when gauge-field fluctuations are very strong, we may expect deviations in the observables due to the 

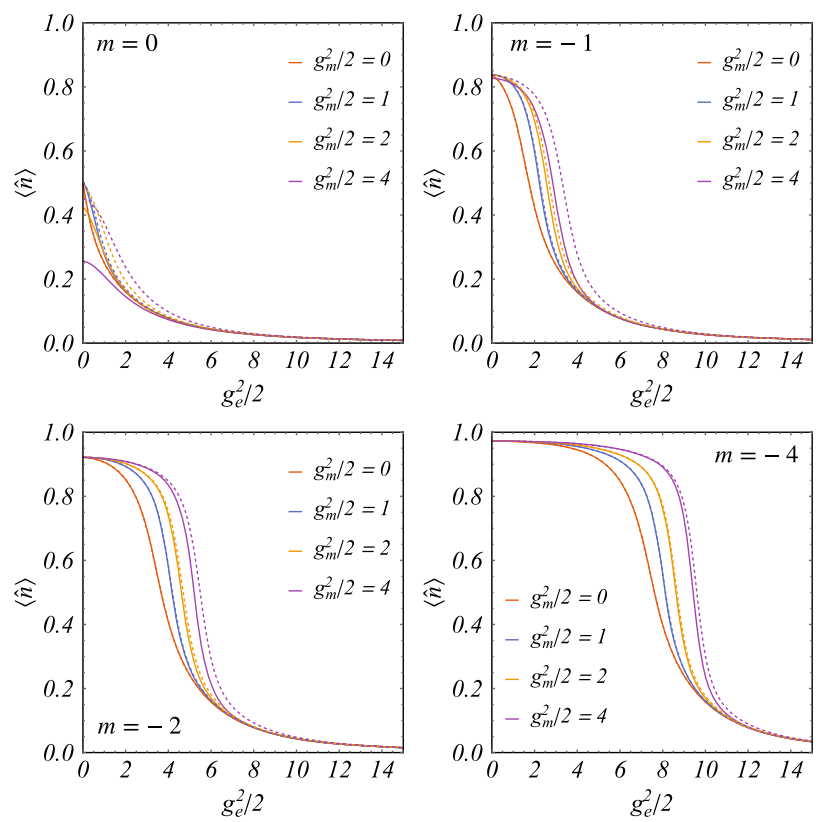

FIG. 20. Particle density as a function of the electric coupling $g_{e}$ for different values of the magnetic coupling $g_{m}$. The four panels represent different bare masses. Solid lines are the $S=1$ results; dashed lines are the $S=2$ results.

finite spin representation of the electric field; in order to have an estimate of the finite- $S$ representation accuracy, we further analyze the $2 \times 2$ plaquette system in the $S=2$ compact representation, namely, when the electric field (in units of flux) can have the values $\{-2,-1,0,1,2\}$. The full Hamiltonian still preserves the block structure in Eq. (I1), where now each mass sector acquires further gauge-invariant states, for a total of five states in the zero-mass sector; 16 states in the $2 m$-mass sector; four states in the $4 m$-mass sector.

As a matter of fact, when $S=2$, the phenomenon of density suppression depicted in Fig. 19 occurs for much larger values of the magnetic couplings, thus disappearing in the limit $S \rightarrow \infty$.

In Fig. 20, we compare the matter density for the two compact representations $S=1,2$ and different values of the couplings. As expected, for $g_{e}^{2} \gg g_{m}^{2}$, the two representations are equivalent; moreover, if $-m \gg 1$ (i.e., very negative) the diagonal configurations are more energetically favorable, and even for small electric coupling and a finite value of $g_{m}^{2}$, the truncation of the gauge-field representation does not affect the results too much $(S=1$ and $S=2$ are almost identical indeed); of course, for $m \geq 0$, this is not the case, and we need $g_{e}^{2} \gg g_{m}^{2}$. Notice that, in the actual QED, this condition is satisfied as long as the electric coupling is sufficiently large since $g_{e}^{2} \sim g_{m}^{-2} \sim g^{2}$.

[1] E. Zohar, J. I. Cirac, and B. Reznik, Simulating Compact Quantum Electrodynamics with Ultracold Atoms: Probing
Confinement and Nonperturbative Effects, Phys. Rev. Lett. 109, 125302 (2012).

[2] D. Banerjee, M. Dalmonte, M. Müller, E. Rico, P. Stebler, U.-J. Wiese, and P. Zoller, Atomic Quantum Simulation of Dynamical Gauge Fields Coupled to Fermionic Matter: From String Breaking to Evolution after a Quench, Phys. Rev. Lett. 109, 175302 (2012).

[3] S. P. Jordan, K. S. M. Lee, and J. Preskill, Quantum Algorithms for Quantum Field Theories, Science 336, 1130 (2012).

[4] L. Tagliacozzo, A. Celi, A. Zamora, and M. Lewenstein, Optical Abelian Lattice Gauge Theories, Ann. Phys. (Amsterdam) 330, 160 (2013).

[5] D. Banerjee, M. Bögli, M. Dalmonte, E. Rico, P. Stebler, U.-J. Wiese, and P. Zoller, Atomic Quantum Simulation of $\mathrm{U}(n)$ and $\mathrm{SU}(n)$ Non-Abelian Lattice Gauge Theories, Phys. Rev. Lett. 110, 125303 (2013).

[6] E. Zohar, J. I. Cirac, and B. Reznik, Cold-Atom Quantum Simulator for SU(2) Yang-Mills Lattice Gauge Theory, Phys. Rev. Lett. 110, 125304 (2013).

[7] L. Tagliacozzo, A. Celi, P. Orland, M. W. Mitchell, and M. Lewenstein, Simulation of Non-Abelian Gauge Theories with Optical Lattices, Nat. Commun. 4, 2615 (2013).

[8] A. Mezzacapo, E. Rico, C. Sabín, I. L. Egusquiza, L. Lamata, and E. Solano, Non-Abelian SU(2) Lattice Gauge Theories in Superconducting Circuits, Phys. Rev. Lett. 115, 240502 (2015).

[9] U.-J. Wiese, Ultracold Quantum Gases and Lattice Systems: Quantum Simulation of Lattice Gauge Theories, Ann. Phys. (N.Y.) 525, 777 (2013).

[10] E. Zohar, J. I. Cirac, and B. Reznik, Quantum Simulations of Lattice Gauge Theories Using Ultracold Atoms in Optical Lattices, Rep. Prog. Phys. 79, 014401 (2015).

[11] E. A. Martinez, C. A. Muschik, P. Schindler, D. Nigg, A. Erhard, M. Heyl, P. Hauke, M. Dalmonte, T. Monz, P. Zoller, and R. Blatt, Real-Time Dynamics of Lattice Gauge Theories with a Few-Qubit Quantum Computer, Nature (London) 534, 516 (2016).

[12] M. C. Bañuls, R. Blatt, J. Catani, A. Celi, J. I. Cirac, M. Dalmonte, L. Fallani, K. Jansen, M. Lewenstein, S. Montangero, C. A. Muschik, B. Reznik, E. Rico, L. Tagliacozzo, K. V. Acoleyen, F. Verstraete, U. J. Wiese, M. Wingate, J. Zakrzewski, and P. Zoller, Simulating Lattice Gauge Theories within Quantum Technologies, arXiv:1911.00003.

[13] M. C. Bañuls and K. Cichy, Review on Novel Methods for Lattice Gauge Theories, arXiv:1910.00257.

[14] I. J. R. Aitchison and A. J. G. Hey, Gauge Theories in Particle Physics: A Practical Introduction. Vol. 1: From Relativistic Quantum Mechanics to QED (CRC Press, Bristol, England, 2003).

[15] T. Cheng and L. Li, Gauge Theory of Elementary Particle Physics (Oxford University, New York, 2006).

[16] J. Kogut and L. Susskind, Hamiltonian Formulation of Wilson's Lattice Gauge Theories, Phys. Rev. D 11, 395 (1975).

[17] E. Fradkin, Field Theories of Condensed Matter Physics, 2nd ed. (Cambridge University Press, Cambridge, England, 2013). 
[18] N. Brambilla et al., QCD and Strongly Coupled Gauge Theories: Challenges and Perspectives, Eur. Phys. J. C 74, 2981 (2014).

[19] J. Schwinger, Gauge Invariance and Mass. II, Phys. Rev. 128, 2425 (1962).

[20] K. G. Wilson, Confinement of Quarks, Phys. Rev. D 10, 2445 (1974).

[21] J. B. Kogut, An Introduction to Lattice Gauge Theory and Spin Systems, Rev. Mod. Phys. 51, 659 (1979).

[22] J. B. Kogut, The Lattice Gauge Theory Approach to Quantum Chromodynamics, Rev. Mod. Phys. 55, 775 (1983).

[23] C. J. Hamer, Z. Weihong, and J. Oitmaa, Series Expansions for the Massive Schwinger Model in Hamiltonian Lattice Theory, Phys. Rev. D 56, 55 (1997).

[24] M. Troyer and U.-J. Wiese, Computational Complexity and Fundamental Limitations to Fermionic Quantum Monte Carlo Simulations, Phys. Rev. Lett. 94, 170201 (2005).

[25] T. M. R. Byrnes, P. Sriganesh, R. J. Bursill, and C. J. Hamer, Density Matrix Renormalization Group Approach to the Massive Schwinger Model, Phys. Rev. D 66, 013002 (2002).

[26] P. Silvi, E. Rico, T. Calarco, and S. Montangero, Lattice Gauge Tensor Networks, New J. Phys. 16, 103015 (2014).

[27] T. Pichler, M. Dalmonte, E. Rico, P. Zoller, and S. Montangero, Real-Time Dynamics in U(1) Lattice Gauge Theories with Tensor Networks, Phys. Rev. X 6, 011023 (2016).

[28] M. Dalmonte and S. Montangero, Lattice Gauge Theory Simulations in the Quantum Information Era, Contemp. Phys. 57, 388 (2016).

[29] M. C. Bañuls, K. Cichy, J. I. Cirac, K. Jansen, and H. Saito, Matrix Product States for Lattice Field Theories, Proc. Sci. LATTICE 2013 (2013).

[30] M. C. Bañuls, K. Cichy, J. I. Cirac, K. Jansen, and S. Kühn, Tensor Networks and their Use for Lattice Gauge Theories, Proc. Sci. LATTICE 2018 (2018).

[31] P. Silvi, Y. Sauer, F. Tschirsich, and S. Montangero, Tensor Network Simulation of an SU(3) Lattice Gauge Theory in 1D, Phys. Rev. D 100, 074512 (2019).

[32] S. Montangero, Introduction to Tensor Network Methods (Springer, Cham, 2018).

[33] L. Funcke, K. Jansen, and S. Kühn, Topological Vacuum Structure of the Schwinger Model with Matrix Product States, Phys. Rev. D 101, 054507 (2020).

[34] F. Bruckmann, K. Jansen, and S. Kühn, O(3) Nonlinear Sigma Model in $1+1$ Dimensions with Matrix Product States, Phys. Rev. D 99, 074501 (2019).

[35] L. Tagliacozzo, A. Celi, and M. Lewenstein, Tensor Networks for Lattice Gauge Theories with Continuous Groups, Phys. Rev. X 4, 041024 (2014).

[36] B. Buyens, J. Haegeman, K. Van Acoleyen, H. Verschelde, and F. Verstraete, Matrix Product States for Gauge Field Theories, Phys. Rev. Lett. 113, 091601 (2014).

[37] E. Rico, T. Pichler, M. Dalmonte, P. Zoller, and S. Montangero, Tensor Networks for Lattice Gauge Theories and Atomic Quantum Simulation, Phys. Rev. Lett. 112, 201601 (2014).
[38] J. Haegeman, K. Van Acoleyen, N. Schuch, J. I. Cirac, and F. Verstraete, Gauging Quantum States: From Global to Local Symmetries in Many-Body Systems, Phys. Rev. X 5, 011024 (2015).

[39] P. Silvi, E. Rico, M. Dalmonte, F. Tschirsich, and S. Montangero, Finite-Density Phase Diagram of a $(1+1)-$ $d$ Non-Abelian Lattice Gauge Theory with Tensor Networks, Quantum 1, 9 (2017).

[40] M. C. Bañuls, K. Cichy, J. I. Cirac, K. Jansen, and S. Kühn, Density Induced Phase Transitions in the Schwinger Model: A Study with Matrix Product States, Phys. Rev. Lett. 118, 071601 (2017).

[41] P. Sala, T. Shi, S. Kühn, M. C. Bañuls, E. Demler, and J. I. Cirac, Gaussian States for the Variational Study of $(1+1)$ Dimensional Lattice Gauge Models," arXiv:1811.04899.

[42] A. Polyakov, Quark Confinement and Topology of Gauge Theories, Nucl. Phys. B120, 429 (1977).

[43] T. Banks, R. Myerson, and J. Kogut, Phase Transitions in Abelian Lattice Gauge Theories, Nucl. Phys. B129, 493 (1977).

[44] S. D. Drell, H. R. Quinn, B. Svetitsky, and M. Weinstein, Quantum Electrodynamics on a Lattice: A Hamiltonian Variational Approach to the Physics of the Weak-Coupling Region, Phys. Rev. D 19, 619 (1979).

[45] S. Ben-Menahem, Confinement in Compact QED for Low Couplings, Phys. Rev. D 20, 1923 (1979).

[46] H. Weimer, M. Müller, I. Lesanovsky, P. Zoller, and H. P. Büchler, A Rydberg Quantum Simulator, Nat. Phys. 6, 382 (2010).

[47] E. Zohar and J. I. Cirac, Combining Tensor Networks with Monte Carlo Methods for Lattice Gauge Theories, Phys. Rev. D 97, 034510 (2018).

[48] F. Tschirsich, S. Montangero, and M. Dalmonte, Phase Diagram and Conformal String Excitations of Square Ice using Gauge Invariant Matrix Product States, SciPost Phys. 6, 28 (2019).

[49] A. Celi, B. Vermersch, O. Viyuela, H. Pichler, M. D. Lukin, and P. Zoller, Emerging 2D Gauge Theories in Rydberg Configurable Arrays, Phys. Rev. X 10, 021057 (2020).

[50] Y.-P. Huang, D. Banerjee, and M. Heyl, Dynamical Quantum Phase Transitions in U(1) Quantum Link Models, Phys. Rev. Lett. 122, 250401 (2019).

[51] M. Gerster, P. Silvi, M. Rizzi, R. Fazio, T. Calarco, and S. Montangero, Unconstrained Tree Tensor Network: An Adaptive Gauge Picture for Enhanced Performance, Phys. Rev. B 90, 125154 (2014).

[52] D. Horn, Finite Matrix Models with Continuous Local Gauge Invariance, Phys. Lett. B 100, 149 (1981).

[53] P. Orland and D. Rohrlich, Lattice Gauge Magnets: Local Isospin From Spin, Nucl. Phys. B338, 647 (1990).

[54] S. Chandrasekharan and U.-J. Wiese, Quantum Link Models: A Discrete Approach to Gauge Theories, Nucl. Phys. B492, 455 (1997).

[55] R. Brower, S. Chandrasekharan, and U.-J. Wiese, QCD as a Quantum Link Model, Phys. Rev. D 60, 094502 (1999).

[56] R. J. Goldston and P. H. Rutherford, Introduction to Plasma Physics (CRC Press: Taylor \& Francis, London, 1995). 
[57] I. Bloch, J. Dalibard, and W. Zwerger, Many-Body Physics with Ultracold Gases, Rev. Mod. Phys. 80, 885 (2008).

[58] T. Hartung and K. Jansen, Zeta-Regularized Vacuum Expectation Values, J. Math. Phys. (N.Y.) 60, 093504 (2019).

[59] F. M. Surace, P. P. Mazza, G. Giudici, A. Lerose, A. Gambassi, and M. Dalmonte, Lattice Gauge Theories and String Dynamics in Rydberg Atom Quantum Simulators, Phys. Rev. X 10, 021041 (2020).

[60] S. Notarnicola, M. Collura, and S. Montangero, Real Time Dynamics Quantum Simulation of $(1+1)$-D Lattice QED with Rydberg Atoms, Phys. Rev. Research 2, 013288 (2020).

[61] M. Bañuls, K. Cichy, J. Cirac, and K. Jansen, The Mass Spectrum of the Schwinger Model with Matrix Product States, J. High Energy Phys. 11 (2013) 158.

[62] E. Zohar and M. Burrello, Formulation of Lattice Gauge Theories for Quantum Simulations, Phys. Rev. D 91, 054506 (2015).

[63] S. Notarnicola, E. Ercolessi, P. Facchi, G. Marmo, S. Pascazio, and F. V. Pepe, Discrete Abelian Gauge Theories for Quantum Simulations of QED, J. Phys. A 48, 30FT01 (2015).

[64] E. Ercolessi, P. Facchi, G. Magnifico, S. Pascazio, and F. V. Pepe, Phase Transitions in $Z_{n}$ Gauge Models: Towards Quantum Simulations of the Schwinger-Weyl QED, Phys. Rev. D 98, 074503 (2018).

[65] P. Corboz, G. Evenbly, F. Verstraete, and G. Vidal, Simulation of Interacting Fermions with Entanglement Renormalization, Phys. Rev. A 81, 010303(R) (2010).

[66] P. Corboz, R. Orús, B. Bauer, and G. Vidal, Simulation of Strongly Correlated Fermions in Two Spatial Dimensions with Fermionic Projected Entangled-Pair States, Phys. Rev. B 81, 165104 (2010).

[67] C. V. Kraus, N. Schuch, F. Verstraete, and J. I. Cirac, Fermionic Projected Entangled Pair States, Phys. Rev. A 81, 052338 (2010).

[68] I. Pižorn and F. Verstraete, Fermionic Implementation of Projected Entangled Pair States Algorithm, Phys. Rev. B 81, 245110 (2010).

[69] Q.-Q. Shi, S.-H. Li, J.-H. Zhao, and H.-Q. Zhou, Graded Projected Entangled-Pair State Representations and an Algorithm for Translationally Invariant Strongly Correlated Electronic Systems on Infinite-Size Lattices in Two Spatial Dimensions, arXiv:0907.5520.

[70] T. Barthel, C. Pineda, and J. Eisert, Contraction of Fermionic Operator Circuits and the Simulation of Strongly Correlated Fermions, Phys. Rev. A 80, 042333 (2009).

[71] C. Pineda, T. Barthel, and J. Eisert, Unitary Circuits for Strongly Correlated Fermions, Phys. Rev. A 81, 050303 (R) (2010).

[72] M. Gerster, M. Rizzi, P. Silvi, M. Dalmonte, and S. Montangero, Fractional Quantum Hall Effect in the Interacting Hofstadter Model via Tensor Networks, Phys. Rev. B 96, 195123 (2017).

[73] M. Collura, L. Dell'Anna, T. Felser, and S. Montangero, On the Descriptive Power of Neural-Networks as Constrained Tensor Networks with Exponentially Large Bond Dimension, arXiv:1905.11351.
[74] P. Silvi, F. Tschirsich, M. Gerster, J. Jünemann, D. Jaschke, M. Rizzi, and S. Montangero, The Tensor Networks Anthology: Simulation Techniques for Many-Body Quantum Lattice Systems, SciPost Phys. Lect. Notes 8 (2019).

[75] I. P. McCulloch, From Density-Matrix Renormalization Group to Matrix Product States, J. Stat. Mech. (2007) P10014.

[76] S. Singh and G. Vidal, Global Symmetries in Tensor Network States: Symmetric Tensors Versus Minimal Bond Dimension, Phys. Rev. B 88, 115147 (2013).

[77] P. Kasteleyn, The Statistics of Dimers on a Lattice: I. The Number of Dimer Arrangements on a Quadratic Lattice, Physica 27, 1209 (1961).

[78] K. Fredenhagen and M. Marcu, Confinement Criterion for QCD with Dynamical Quarks, Phys. Rev. Lett. 56, 223 (1986).

[79] K. Gregor, D. A. Huse, and R. Moessner, and S. L. Sondhi, Diagnosing Deconfinement and Topological Order, New J. Phys. 13, 025009 (2011).

[80] E. Zohar, M. Burrello, T. B. Wahl, and J. I. Cirac, Fermionic Projected Entangled Pair States and Local U(1) Gauge Theories, Ann. Phys. (Amsterdam) 363, 385 (2015).

[81] R. Orús, Tensor Networks for Complex Quantum Systems, Nat. Rev. Phys. 1, 538 (2019).

[82] U. Schollwöck, The Density-Matrix Renormalization Group in the Age of Matrix Product States, Ann. Phys. (Amsterdam) 326, 96 (2011).

[83] F. Verstraete, M. M. Wolf, D. Perez-Garcia, and J. I. Cirac, Criticality, the Area Law, and the Computational Power of Projected Entangled Pair States, Phys. Rev. Lett. 96, 220601 (2006).

[84] F. Verstraete and J. I. Cirac, Matrix Product States Represent Ground States Faithfully, Phys. Rev. B 73, 094423 (2006).

[85] S. Östlund and S. Rommer, Thermodynamic Limit of Density Matrix Renormalization, Phys. Rev. Lett. 75, 3537 (1995).

[86] F. Verstraete and J. I. Cirac, Valence-Bond States for Quantum Computation, Phys. Rev. A 70, 060302(R) (2004).

[87] R. Orús, A Practical Introduction to Tensor Networks: Matrix Product States and Projected Entangled Pair States, Ann. Phys. (Amsterdam) 349, 117 (2014).

[88] Y.-Y. Shi, L.-M. Duan, and G. Vidal, Classical Simulation of Quantum Many-Body Systems with a Tree Tensor Network, Phys. Rev. A 74, 022320 (2006).

[89] P. Silvi, V. Giovannetti, S. Montangero, M. Rizzi, J. I. Cirac, and R. Fazio, Homogeneous Binary Trees as Ground States of Quantum Critical Hamiltonians, Phys. Rev. A 81, 062335 (2010).

[90] G. Vidal, Entanglement Renormalization, Phys. Rev. Lett. 99, 220405 (2007).

[91] G. Evenbly and G. Vidal, Entanglement Renormalization in Two Spatial Dimensions, Phys. Rev. Lett. 102, 180406 (2009).

[92] S. R. White, Density Matrix Formulation for Quantum Renormalization Groups, Phys. Rev. Lett. 69, 2863 (1992).

[93] S. R. White and A. E. Feiguin, Real-Time Evolution Using the Density Matrix Renormalization Group, Phys. Rev. Lett. 93, 076401 (2004). 
[94] G. Vidal, Efficient Classical Simulation of Slightly Entangled Quantum Computations, Phys. Rev. Lett. 91, 147902 (2003).

[95] J. Haferkamp, D. Hangleiter, J. Eisert, and M. Gluza, Contracting Projected Entangled Pair States is Average-Case Hard, Phys. Rev. Research 2, 013010 (2020).

[96] A. J. Ferris, Area Law and Real-Space Renormalization, Phys. Rev. B 87, 125139 (2013).

[97] T. D. Kühner, S. R. White, and H. Monien, One-Dimensional Bose-Hubbard Model with Nearest-Neighbor Interaction, Phys. Rev. B 61, 12474 (2000).
[98] S.-J. Gu, Fidelity Approach to Quantum Phase Transitions, Int. J. Mod. Phys. B 24, 4371 (2010).

[99] B. Damski, Fidelity Susceptibility of the Quantum Ising Model in a Transverse Field: The Exact Solution, Phys. Rev. E 87, 052131 (2013).

[100] D. Braun, G. Adesso, F. Benatti, R. Floreanini, U. Marzolino, M. W. Mitchell, and S. Pirandola, QuantumEnhanced Measurements without Entanglement, Rev. Mod. Phys. 90, 035006 (2018).

[101] D. Rossini and E. Vicari, Ground-State Fidelity at FirstOrder Quantum Transitions, Phys. Rev. E 98, 062137 (2018). 